Rev. Elev. Méd. Vét. Pays trop., 1966, 19, 3 (283-306).

\title{
Action d'un nouvel anthelminthique le tétramisole (16.535 R.P.) sur divers helminthes du mouton de la République du Tchad ${ }^{*}$
}

\author{
por M. GRABER
}

\begin{abstract}
RÉSUMÉ
L'auteur, sur 259 ovins de la République du Tchad, étudie le pouvorr anthelminthique d'un nouvecu dérivé de l'Imidazole, le Tétramisole (16.535 R. P.).

Le médicament, s'il est totalement inaclif sur les Trématodes et les Cestodes de l'appareil digestif, assure par contre, aux doses de $40 \mathrm{mg} / \mathrm{kg}$ par la voie buccale ou de $12 \mathrm{mg} / \mathrm{kg}$ par la voie sous-cutanée (en solution à $10 \mathrm{p}$. 100), la destruction à peu près complère des espèces parasites suivantes : Gaigerio pachyscelis, Haemoncus contortitus, Oesophogostomum columbianum adultes mûrs ou immatures. L'action sur Stongyloides adultes ou immatures est moins bonne; sur Buckleyuris ovis et sur Buckleyuris globulasa, elle semble irrégulière, mais supérieure au Thiabendazole. Beaucoup de larves $L_{4}$ intranodulaires d'Oesophagostomum columbionum résistent au traitement, quelle que soit la dose utilisée.

Le Tétramisole qui se comporte comme un nématodifuge présente une valeur économique certaine, puisque, sur le terrain au bout de cinq semaines, les gains de poids sont de l'ordre de $10,9-13,7$ p. 100.

Le Coefficient chimiatherrapique varie de 4,5 à 5 pour la voie buccale el 5 à 5,4 pour la voie sous-cutanée. La toxicité du 16.535 R. P. pour le mouton tchadien est donc supérieure à celle du Thiabendazole. Aux doses thérapeutiques, la tolérance des femelles gestantes et des agneaux de lait est satisfoisante.
\end{abstract}

\section{INTRODUCTION}

Parmi tous les parasites intestinaux qu'hébergent les ovins de la République du Tchad, les Nématodes tiennent une place de choix. Si l'on considère les chiffres actuellement connus qui portent sur 4.226 autopsies effectuées de 1954 à 1964 (GRABER, 1966), on note la présence dans l'intestin des animaux atteints de Strongyloides papil-

\footnotetext{
* Institut d'Elevage et de Médecine vétérinaire des Pays Tropicaux, Laboratoire de Farcha-Fort-Lamy, République du Tchad. Société Rhone-Poulenc, 22 Avenue Montaigne, Paris.
}

losus (WEDL, 1856). Oesophogostomum columbianum (CURTICE, 1890). Gaigerio pachyscelis (RAILLIET, 1900). Bunostomum trigonocephalum (RUDOLPHI, 1808). Haemoncus contortus (RUDOLPHI, 1803) Haemoncus placel (PLACE, 1893). Buckleyuris globuloso (Von LINSTOW, 1901). Buckleyuris ovis (ALBIGAARD, 1795).

Ces huit espèces sont associées dans 50 p. 100 des cas.

Outre une mortalité élevée, variable selon les années et les régions, les Nématodes de l'intestin entraînent des pertes indirectes importantes, l'ensemble pouvant être estimé à environ 
12 p. 100 de la valeur mercuriale du troupeau (GRABER, 1966).

Aussi, depuis longtemps, la Section d'Helminthologie du Laboratoire de FARCHA a-t-elle étudié les moyens thérapeutiques d̀ mettre en œuvre pour détruire ou tout au moins atténuer l'incidence de ce parasitisme.

De nombreux médicaments ont été utilisés et comparés (Phénothiazine, Béphénıum Thiabendazole, etc...),

Tout récemment, à la demande de la Société Rhône-Poulenc, des essais ont été tentés avec le Tétramisole (16.535 R. P.), nouvel anthelminthique mis au point par JANSSEN Pharmaceutica*.

\section{I. - MATÉRIEL ET MÉTHODE}

\section{A. - L'Anthelminthique.}

Le Tétramisole, nom générique, est le dltetrahydro-2, 3, 5, 6, phenyl-6 imidazo (2, I-b) thiazole. Chlorhydrate de formule $\mathrm{C}_{11} \mathrm{H}_{12} \mathrm{~N}_{2} \mathrm{~S}$, HCL.<smiles>ClC1CC2CC(c3ccccc3)N=C12</smiles>

Le Chlorhydrate titre 84.85 p, 100 de produit actif de base, Son poids moléculaire est de $240,753$.

II se présente sous l'aspect d'une poudre cristalline blanche, inodore, stable, soluble dans

* Beerse-Belgique.
I'eau ( $1 \mathrm{~g}$ dans $4 \mathrm{ml}$ ) et l'éthanol $(1 \mathrm{~g}$ dans $70 \mathrm{ml})$, mais nan dans le chloroforme au l'Acétone.

Le Tétramisole est encore connu sous les noms de Ripercol (Belgique) et de Nilverm (Angleferre) **.

\section{B. - Les animaux d'expérience.}

259 ovins originaires du Chari-Baguirmi et du Kanem (Ovest Tchad) et pesant de 12 à $36 \mathrm{~kg}$ ont été utilisés, se répartissant ainsi :

Essais thérapeutiques proprement dits : 118, Essals de toxicité : 37.

Essais sur le terrain : 20.

Brebis gestantes : 9 .

Agneaux d'un mois : 6 .

Témoins : 69.

La plupart d'entre eux étaient porteurs, à l'état naturel, d'un grand nombre d'Helminthes adultes ou immatures (voir le Tableau):

Ces Parasites se trouvaient associés dans 35 p. 100 des cas. Dans ces conditions. l'étaf général des animaux mis en expérience était des plus médiocres, ce qui a permis de préciser la toxicité du médicament sur un troupeau peu résıstant et mal entretenu.

Les essais ont été menés en quatre étapes de févrıer 1965 à mars 1966 :

Février-mars-avril 1965.

Juin-juillet 1965 .

** Le médicament va être commercialisé incessamment en France par SPECIA et Roger BELLON sans le nom de MEMICINE en solution prête à l'emploi au taux de 7,5 p. 100.

TABLEAU

\begin{tabular}{|c|c|c|c|}
\hline Espèces parasites & Animaux traités & Anzinaux témoins & Total (sur 205 ovins) \\
\hline $\begin{array}{l}\text { Fasciola gigantıca } \\
\text { Paramphistomum nicrobothrium } \\
\text { Camyerius papillatus } \\
\text { Schistosoma bovis } \\
\text { Moniezia expansa } \\
\text { Stilesia hepatica } \\
\text { Stilesia globipunctata } \\
\text { Avitelling centripunctata } \\
\text { Avitellina woodlandi } \\
\text { Strongyloides papil.losus } \\
\text { Gaigerıa pachyscelis } \\
\text { Oesophagostoulum columbianum } \\
\text { (aduites ) } \\
\text { Oesophagostones nodulanres } \\
\text { Haemoncus contortus } \\
\text { Buckleynuris ovis } \\
\text { Buckleyuris globulosa }\end{array}$ & $\begin{array}{r}36 \\
1 \\
36 \\
20 \\
22 \\
80 \\
\\
45 \\
118 \\
17 \\
78 \\
39 \\
53 \\
9\end{array}$ & $\begin{array}{l}1 \\
8 \\
11 \\
4 \\
3 \\
35 \\
20 \\
22 \\
10 \\
37 \\
21 \\
21 \\
7\end{array}$ & $\begin{array}{r}1 \\
44 \\
1 \\
47 \\
24 \\
25 \\
115 \\
65 \\
65 \\
140 \\
27 \\
115 \\
60 \\
74 \\
\\
16\end{array}$ \\
\hline
\end{tabular}


Octobre-novembre-décembre 1965. Février-mars 1966.

II est ainsi possible d'apprécier, après traitement, le comportement des moutons auxépoques favorables ou' les conditions alimentaires sont relativement satisfaisantes de (septembre à mars) et aux époques moins favorables (d'avril à aoôt). Par ailleurs, compte tenu de ce que l'on connaît de la biologie des Nématodes ovins en République du Tchad (GRABER, 1965), cette façon d'opérer donne des indications sur le pouvoir anthelminthique réel du Tétramisole à l'égard des Nématodes saisonniers (Hoemoncus contortus en hivernage, de juin à octobre) et de ceux dont l'évolution est plus longue et plus étalée dans le temps (Oesophagostomum columbianum notamment).

\section{C. - Méthode.}

Le protocole d'expérience est demeuré très classique (GRABER, 1965 a). It comprend plusieurs séries d'opérations :

\section{Avant traitement.}

Chaque animal est mis en observation pendant 48 heures ef divers examens (coproscopiques et hématologiques) sont pratiqués dans le but de situer l'importance exacte du parasitisme. Parallèlement, des cultures d'œufs permettent de savoir à quels Némaiodes on a affaire. En fonction des renseignements cinsi obtenus, les lots sont constitués : ils comprennent un tiers d'animaux très parasités. un tiers d'animaux moyennement parasités et un tiers d'animaux faiblement parasités.

\section{Traifement.}

La totalité des essais a été effectuée sans préparation, c'est-à-dire sans diète préalable : le traitement des animaux sur le terraın, du fait de la mentalité des éleveurs, ne doit, en effet, comporter aucune préparation particulière de l'animal.

Les animaux ont été marqués ef placés dans des stalles individuelles cimentées.

L'anthelminthique a été administré de deux manières :

- par la voie buccale « à la bouteille 》.

- par la voie sous-cutanée. Le 16.535 R. P. est alors dissous dans de l'eau distillée (solution d̀ 10 p. 100) maintenue une demi-heure à $30^{\circ} \mathrm{C}$, de façon à permettre la disparition des derniers cristaux visibles. Quand la solution est absolument claire, sans dépôt, le médicament est injecté derrière l'époule. L'inoculation est bien supportée et, à l'autopsie, au bout de 10-15 jours, aucune trace de nécrose n'a été observée au point d'inoculation.

Que ce soit par la voie buccale ou par la voie sous-cutanée, les doses distribuées sont des doses calculées en produit de base pur. Le médicament étant sous forme de Chlorhydrate, une correction est nécessaire (1 g de produit pur correspond à $1,1765 \mathrm{~g}$ de (hlorhydrate),

\section{$3^{\circ}$ Après troitement.}

Sur chaque animal, il a été procédé pendant 8 à 10 jours' :

a) au prélèvement des crottes trois fois par jour. Elles ont été broyées dans un mince filet d'eau et soigneusement examinées, de manière à faire apparaître les Helminthes-Cestodes et Nématodes - évacués après l'administration du 16.535 R. P. Ceux-ci ont été pesés, comptés et déterminés.

b) A des examens coproscopiques journaliers par la méthode de sédimentation de BRUMPT, la plus simple à meitre en cuvre dans ce pays. La comparaison entre la moyenne du nombre d'œufs au gramme avant traitement, après traitement ef le jour de l'autopsie permet d'avoir un premier aperçu de l'efficaciłé du médicament.

c) A des cultures d'œufs en boîtes de Pétri sur papier buvard humide. Arbitrairement, on évalue chaque jour le nombre de larves $L_{3}$ 'rencontrées dan's une goutte de suspension aqueuse provenant des boîtes de Pétri. Pour chaque lot, la comparaison entre la moyenne du nombre de larves avant et après le traitement complète en général les éléments d'appréciation fournis par l'examen coproscopique. Pour certains Nématodes digérés dans l'intestin à la suite du traitement au Tétramisole, la méthode est absolument indispensable (Strongyloides ef Hoemoncus).

\section{Autopsie.}

Passé ce délai, les animaux sont sacrifiés. Les Helminthes demeurés en place sọt récoltés : 
ce travail ne pose pas de problème pour les grands Cestodes, les Gaigeria, les Oesophagostomum adultes ef les Trichures. La comparaison entre le nombre (Nématodes) ou le poids (Cestodes et Trématodes) des parasites expulsés et le nombre ou le poids de parasites encore pré. sents dans l'intestin donne le pourcentage d'efficacité.

Lorsqu'il s'agit de petits Nématodes (Strongyloides) ou de Strlesia globupunctata, il importe de gratter la muqueuse duodénale sur une longueur de 30 à $50 \mathrm{~cm}$, L'examen au microscope du produit de raclage placé entre lame et lamelle confirme ou non l'existence de Strongyloides papillosus ou de scolex de Stilesia globipunctata. Pour ce dernier Cestode, la comparaison entre le nombre total de nodules et le nombre de scolex trouvés fait connaitre le pouvoir anthelminthique du Tétramisole à l'égard de ce parasite.

\section{II. - RÉSULTATS}

\section{A. - Les témoin's (Tableau no 1).}

\section{B. - Action sur les trématodes.}

Aux doses utilisées, quel que soit le mode d'administration, le Tétramisole est dépourvu de toute activité à l'égard de Paramphistomum microbothrium et de Carmyerius papillatus de la panse, ainsi que de Schistosoma bovis des veines hépatıques et mésentériques (Tableau no Ii).

\section{C. - Action sur les cestodes (Tableau na III).}

Même à très fortes doses, le Tétramisole est totalement inefficace sur les Anoplocephalidae de l'intestin (Moniezia expansa, Stilesia globipunctata. Avitellina centripunctata et Avitellina woodlandi) ef des canaux biliaires (Stilesia hepatica).

\section{D. - Action sur les nématodes.}

10 Sur les formes adultes mûres.

Tableau no IV : examens coproscopiques.

Tableau $n^{\circ} V$ : Strongyloides papillosus.

Tableau no VI : Gaigeria pachyscelis.

Tableav no VII : Oesophagostomum columbianum.

Tableau no VIII : Hoemoncus contortus.

Tableau no IX: Buckleyuris ovis et Buckleyuris globulosa.

$2^{0}$ Sur les formes adultes immatures d'Oesophagostomum columbianum et sur les larves $L_{4}$ intranodulaires du même parasite.

Au Tchad, on sait (GRABER et RECEVEUR, 1956 ; GRABER 1965 b) qu'Oesophogostomum colombianum adulte se rencontre dans l'intestin du mouton à des époques de l'année bien déterminées :

\begin{tabular}{|c|c|c|c|c|c|c|c|c|}
\hline \multicolumn{9}{|c|}{$\begin{array}{l}\text { TABLEAJ NOI } \\
\text { Témoing }\end{array}$} \\
\hline Espèces en cause & $\begin{array}{c}\text { Fétrier } \\
1965 \\
\left(8^{+}\right)\end{array}$ & $\begin{array}{r}\text { Mars } \\
1965 \\
(8)\end{array}$ & $\begin{array}{l}\text { Avril } \\
1965 \\
(12)\end{array}$ & $\begin{array}{c}\text { Mat } \\
\text { Juin } \\
1965(6)\end{array}$ & $\begin{array}{c}\text { Detobre } \\
1965 \\
(9)\end{array}$ & $\begin{array}{c}\text { Novembre } \\
1965 \\
(7)\end{array}$ & $\begin{array}{c}\text { Décembre } \\
1965 \\
\text { (11) }\end{array}$ & $\begin{array}{c}\text { Février } \\
1966 \\
\text { (B) }\end{array}$ \\
\hline 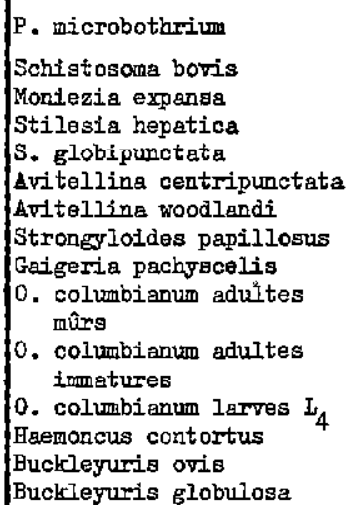 & $\begin{array}{l}0,8^{++} \\
28^{+++} \\
- \\
1 \\
- \\
2^{+++} \\
4 \\
8 \\
22 \\
2 \\
4 \\
1\end{array}$ & $\begin{array}{l}2 \\
- \\
- \\
0,8 \\
- \\
4 \\
1 \\
26 \\
11 \\
1 \\
- \\
-\end{array}$ & $\begin{array}{l}21 \\
- \\
1,4 \\
2,4 \\
5 \\
10 \\
1 \\
15 \\
2 \\
2 \\
6 \\
1\end{array}$ & $\begin{array}{l}2,2 \\
2 \\
= \\
1,7 \\
12 \\
= \\
23 \\
23 \\
25 \\
4\end{array}$ & $\begin{array}{c}5 \\
- \\
1,6 \\
14 \\
2 \\
- \\
- \\
- \\
2 \\
41 \\
-\end{array}$ & $\begin{array}{l}1,05 \\
5 \\
= \\
1 \\
- \\
5 \\
4 \\
- \\
= \\
4 \\
- \\
-\end{array}$ & $\begin{array}{l}1 \\
6 \\
3,5^{+1} \\
1,5 \\
1,4 \\
8,6 \\
4 \\
6 \\
3 \\
5 \\
7 \\
7 \\
-\end{array}$ & $\begin{array}{c}1 \\
2 \\
2 \\
- \\
1,5 \\
13,5 \\
31 \\
- \\
25 \\
12 \\
- \\
10 \\
14\end{array}$ \\
\hline
\end{tabular}

+ Nombre de moutons utilisés

++ Cestodes et Trématode日 : poida mojen de paragites (én g.)

++ Sahistosomes et Nématodes : moyenne du nombre de parasites. 
TABIEAU NOII

Trématodes-Nombre d'animaux déparesités après traitument au Tétramisole

\begin{tabular}{|c|c|c|c|c|}
\hline $\begin{array}{l}\text { Doses } \\
\mathrm{mg} / \mathrm{kg}\end{array}$ & $\begin{array}{l}\text { Fasciola } \\
\text { glgantica }\end{array}$ & $\begin{array}{l}\text { Paramphistomum } \\
\text { microbothrium }\end{array}$ & $\begin{array}{l}\text { Carmyerius } \\
\text { pap1llatus }\end{array}$ & $\begin{array}{c}\text { Schistosome } \\
\text { bovi.a }\end{array}$ \\
\hline $\begin{array}{c}\text { Voie b } \\
5 \\
10 \\
20 \\
25 \\
30 \\
35 \\
40 \\
100 \\
150\end{array}$ & $\begin{array}{c}0 \text { sur } \\
- \\
- \\
- \\
- \\
= \\
- \\
-\end{array}$ & $\begin{array}{lll}0 & \text { sur } & 5 \\
0 & \text { sur } & 7 \\
0 & \text { sur } & 2 \\
0 & \text { sur } & 2 \\
0 & \text { sur } 1 \\
0 & \text { sur } & 1 \\
0 & \text { sur } & 4 \\
0 & \text { sur } & 2 \\
0 & \text { sur } & i\end{array}$ & $\begin{array}{c}0 \text { sur } \\
- \\
- \\
= \\
= \\
= \\
= \\
-\end{array}$ & $\begin{array}{lll}0 & \text { sur } & 1 \\
0 & \text { sur } & 2 \\
0 & \text { sur } & 2 \\
0 & \text { sur } & 5 \\
0 & \text { sur } & 2 \\
0 & \text { sur } & 3 \\
0 & \text { sur } & 3 \\
0 & \text { sur } & 1\end{array}$ \\
\hline $\begin{array}{r}\text { Voie } \\
5 \\
12 \\
15 \\
30 \\
70\end{array}$ & $\begin{array}{l}\bar{z} \\
\tilde{z}\end{array}$ & $\begin{array}{c}0 \text { sur } 2 \\
0 \text { sur } 4 \\
0 \text { sur } 5 \\
- \\
-\end{array}$ & $\begin{array}{l}\overline{-} \\
\overline{-} \\
\overline{-}\end{array}$ & 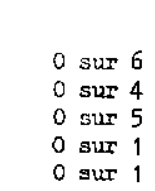 \\
\hline
\end{tabular}

TABLFAU NOIII

Cestodes-Nombre d'animaux déperasités après treitement æ Tétramisole.

\begin{tabular}{|c|c|c|c|c|}
\hline $\begin{array}{l}\text { Doges } \\
\mathrm{Mg} / \mathrm{kg}\end{array}$ & $\begin{array}{l}\text { Moniezia } \\
\text { expanga }\end{array}$ & $\begin{array}{l}\text { St1lesia } \\
\text { hepatica }\end{array}$ & $\begin{array}{l}\text { Stileaia } \\
\text { globipunctata }\end{array}$ & $\begin{array}{l}\text { Avitellina contripunctata } \\
\text { Avitellina woodlandi }\end{array}$ \\
\hline $\begin{array}{c}\text { Voie buccale. } \\
5 \\
10 \\
20 \\
25 \\
30 \\
35 \\
40 \\
100 \\
150\end{array}$ & 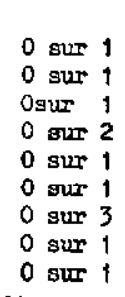 & $\begin{array}{c}- \\
- \\
- \\
0 \text { sur } 3 \\
0 \text { sur } 1 \\
0 \text { sur } 2 \\
0 \text { sur } 4 \\
0 \text { sur } 1\end{array}$ & $\begin{array}{c}0 \text { sur } 2 \\
0 \text { sur } 9 \\
0 \text { sur } 5 \\
0 \text { sur } 7 \\
0 \text { sur } 4 \\
0 \text { sur } 6 \\
0 \text { sur } 8 \\
0 \text { sur } 1 \\
\text { - }\end{array}$ & $\begin{array}{l}0 \text { sur } 3 \\
0 \text { sur } 5 \\
0 \text { sur } 3 \\
0 \text { sur } 2 \\
0 \text { sur } 4 \\
0 \text { sur } 6 \\
0 \text { sur } 3 \\
0 \text { sur } 3\end{array}$ \\
\hline \multicolumn{5}{|c|}{ Voile sous-cutanée } \\
\hline $\begin{array}{r}5 \\
12 \\
15 \\
30 \\
40 \\
50 \\
60 \\
70\end{array}$ & $\begin{array}{lll}0 & \text { sur } & 2 \\
0 & \text { sur } & 1 \\
0 & \text { sur } & 1 \\
0 & \text { sur } & 1 \\
0 & \text { sur } & 1 \\
0 & \text { aur } & 1 \\
0 & \text { sur } & 1\end{array}$ & $\begin{array}{c}0 \text { sur } 4 \\
0 \text { sur } 3 \\
- \\
0 \text { sur } 2 \\
- \\
0 \text { sur } 2 \\
- \\
-\end{array}$ & $\begin{array}{l}0 \text { sur } 5 \\
0 \text { sur } 13 \\
0 \text { sur } 6 \\
0 \text { sur } 6 \\
0 \text { sur } 5 \\
0 \text { sur } 2 \\
0 \text { sur } 1\end{array}$ & 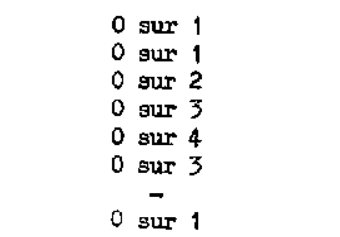 \\
\hline
\end{tabular}

- en saison des pluies (coût-septembre),

- en saison sèche (de mars à juin),

ainsi que l'indique le graphique $n^{0} 1$ valable pour les années 1959-1960 dans la préfecture du Chari-Baguirmi.

Dans le premier cas, la présence d'Oesophagostomes adultes correspond à des primoinfestations du début de la saison des pluies avec cycle normal (VEGLIA, 1923), chez des animaux de l'année qui n'ont pas encore été en contact avec le Nématode.

Dans le second cas, le processus s'apparente à celui décrit par MAROTEL (1908) pour Bostcola rodiatum du bœuf, Il s'agit de réinfestations ou de sur-infestations se produisant en saison des pluies et déclenchant, chez des moutons porteurs d'Oesophagostomes, un processus allergique au niveau de l'intestin avec pénétratıon des larves infestantes en profondeur. II se forme alors des nodules parasitaires renfermant des larves $L_{4}$ bien vivantes. Celles-ci vont séjourner dans les nodules la plus grande partie de la saison sèche et ne passeront dans l'intestin qu'à partir de janvier ou de février, selon les années, 
Graphique NO1' Infestation por Desophogostomum Columbianum CHARI-BAGUIRMI

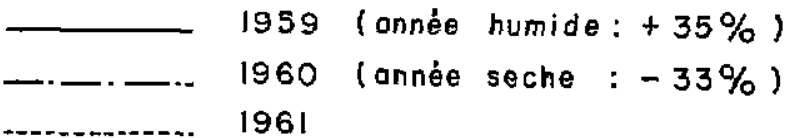

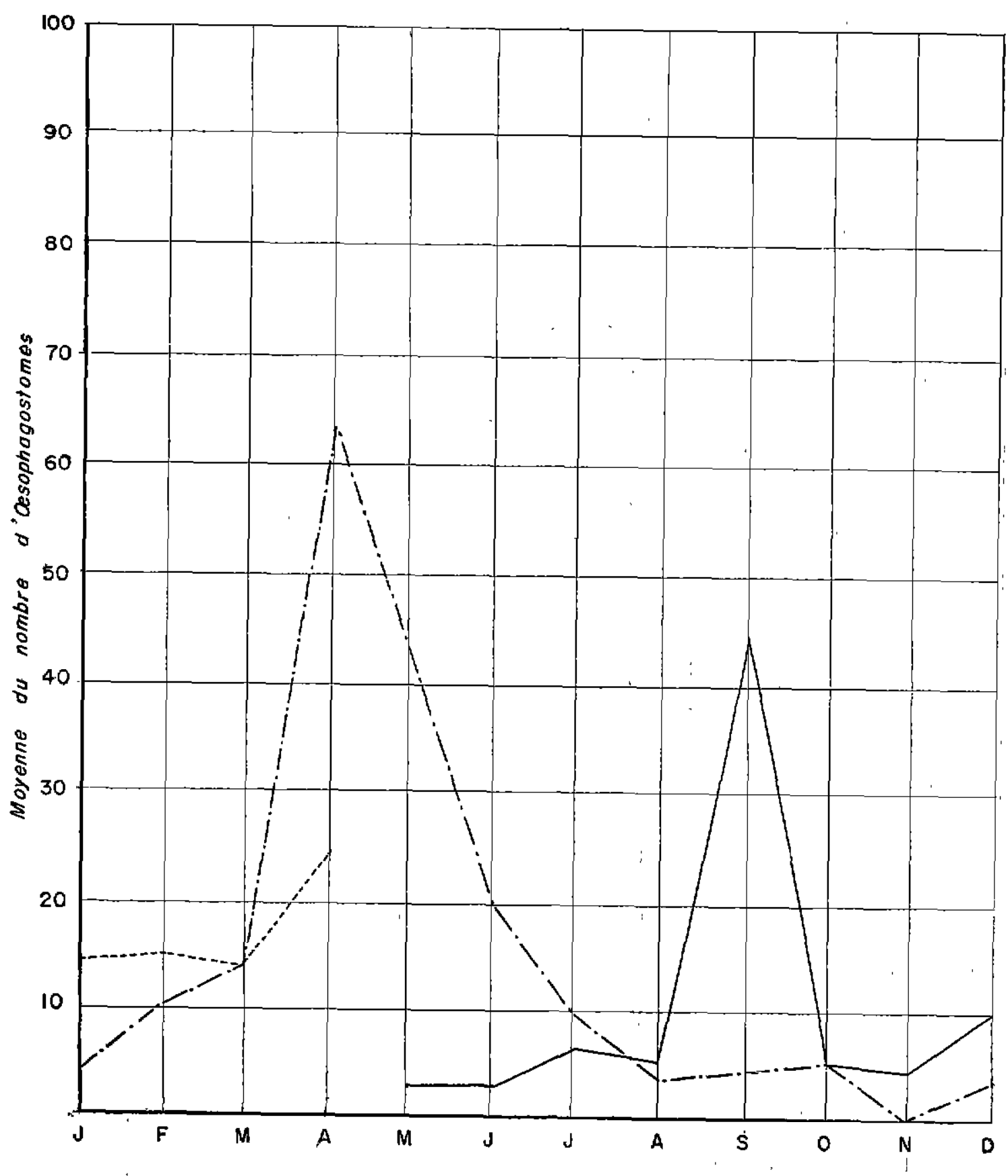


TABLBAD N OIV

Moyenne du nombre d'oeuf's an grame de matière f'écale.

\begin{tabular}{|c|c|c|c|c|c|c|}
\hline \multirow{2}{*}{$\begin{array}{l}\text { Doseg } \\
\mathrm{mg} / \mathrm{kg}\end{array}$} & \multicolumn{2}{|c|}{ Avant traitement } & \multicolumn{2}{|c|}{ Après traitement } & \multicolumn{2}{|c|}{ Dernier jour + } \\
\hline & Strongles & Strongr loides & Strongles & Strongyloides & Strongles & Strongyloides \\
\hline \multicolumn{7}{|c|}{ Vole buccale } \\
\hline $\begin{array}{r}5 \\
10 \\
20 \\
25 \\
30 \\
35 \\
40\end{array}$ & $\begin{array}{r}31 \\
58 \\
70 \\
226 \\
31 \\
- \\
105\end{array}$ & $\begin{array}{c}\overline{-} \\
\overline{60} \\
\overline{124} \\
90\end{array}$ & $\begin{array}{r}0 \\
22 \\
18 \\
130 \\
0 \\
- \\
25\end{array}$ & $\begin{array}{r}- \\
- \\
\overline{0} \\
6 \\
185 \\
40\end{array}$ & $\begin{array}{l}0 \\
0 \\
0 \\
0 \\
0 \\
- \\
0\end{array}$ & $\begin{array}{r}- \\
- \\
- \\
32 \\
0 \\
1 \\
0\end{array}$ \\
\hline \multicolumn{7}{|c|}{ Vore sous-cutané } \\
\hline $\begin{array}{r}5 \\
12 \\
15 \\
30 \\
40\end{array}$ & $\begin{array}{r}12 \\
105 \\
60 \\
5 \\
52\end{array}$ & $\begin{array}{r}14 \\
21 \\
105 \\
36 \\
-\end{array}$ & $\begin{array}{r}5 \\
5 \\
50 \\
5 \\
12\end{array}$ & $\begin{array}{r}17 \\
0 \\
40 \\
10 \\
-\end{array}$ & $\begin{array}{l}6 \\
0 \\
0 \\
0 \\
0\end{array}$ & $\begin{array}{r}18 \\
0 \\
0 \\
0 \\
-\end{array}$ \\
\hline
\end{tabular}

+7 a 10 jours après $1 a$ fin du traitement.

"Strongles" : Oesophagostomum adultes mîrs - Haenoncus - Gaigeria.

"Strongy loides" : Strongy loides papillosus.

Elles subissent peu à peu la maturation sexuelle, Les œufs ne deviennent abondants dans les crottes qu'en mai-juin-juillet, lls tombent sur le sol et, si les conditions extérieures sont favorables, ils donnent naissance en $9-12$ jours à des larves $L_{3}$ susceptibles d'infester d'autres ovins neufs qui utilisent les mêmes parcours, On retombe alors dans le premier cas.

La coexistence des deux cycles assure la pérennité de l'infestation des animaux et des pâtures.

En vue de couper le cycle, des essais ont été tentés pour déterminer l'effet du Tétramisole sur les formes larvaires $L_{4}$ intranodulaires d'Oesophagostomum columbianum et sur les formes adultes, mais immatures du même parasite (Tableaux $n^{\circ} X$ et $X l$ ).

30 Sur les formes immatures de Strongylordes popillosus (Tableau XII).

40 Discussion.

Le Tétramisole.

a) Provoque une forte diminution du nombre d'cufs d'Haemoncus, d'Oesophagostomum columbianum et de Strongyloides popillosus.

b) Assure la destruction à $40 \mathrm{mg} / \mathrm{kg}$ (V. B. *) ou d̀ $12 \mathrm{mg} / \mathrm{kg}$ (V.S.C. ${ }^{* *}$ ) de $94-95$ p. 100 des formes adultes de Strongyloides papillosus et d'un certain nombre de formes larvaires immatures

*V. B. $=$ Voie buccale.

$* *$ V. S. C. $=$ Voie sous-cutanée. du même parasite : dans les deux cas, l'efficacité n'est pas totale et il faut employer des doses élevées pour obtenir la disparition complète du Nématode.

C) Permet vers $10 \mathrm{mg} / \mathrm{kg}$ (V. B.) ou $5 \mathrm{mg} / \mathrm{kg}$ (V.S. C.) l'expulsion de tous les Gaigeria pachyscelis adulte.

d) Les Hoemoncus contortus sont tués à partir de $5 \mathrm{mg} / \mathrm{kg}$, quel que soit le mode d'administration. Les essais n'ayant porté que sur des ovins faiblement ou moyennement parasités, d'autres expériences sont nécessaires pour confirmer cette posologie dans les cas d'infestations massives.

e) L'action sur Buckleyuris ovis ef sur Buckleyuris globuloso est plus irrégulière : elle paraît satisfaisante vers $30 \mathrm{mg} / \mathrm{kg}$ (V. B.).

f) Les Oesophagostomum columbianum adultes mûres ou immatures sont éliminés vers $25 \mathrm{mg} /$ $\mathrm{kg}$ (V. B.) ou $12 \mathrm{mg} / \mathrm{kg}$ (V. S. C.). Quant aux formes larvaires $L_{4} .25$ à 34 p. 100 d'entre elles ne sont pas touchées ef demeurent vivantes dans les nodules intracaecaux.

A titre de comparaison. le Tableau $n^{\circ}$ XIII donne les pourcentages d'efficacité du Thiabendazole (GRABER, 1966) à l'égard des principaux Nématodes ovins recueillis au Tchad (voir graphiques $n(0|f| I,|| V,$,$V et V \mid)$ :

En définitive, le Tétramisole semble supérieur au Thiabendazole, en ce qui concerne son action 
TABLLAU NOV

sction de Tétramiaole gur Strongyloides papillosus aduilte mîr.

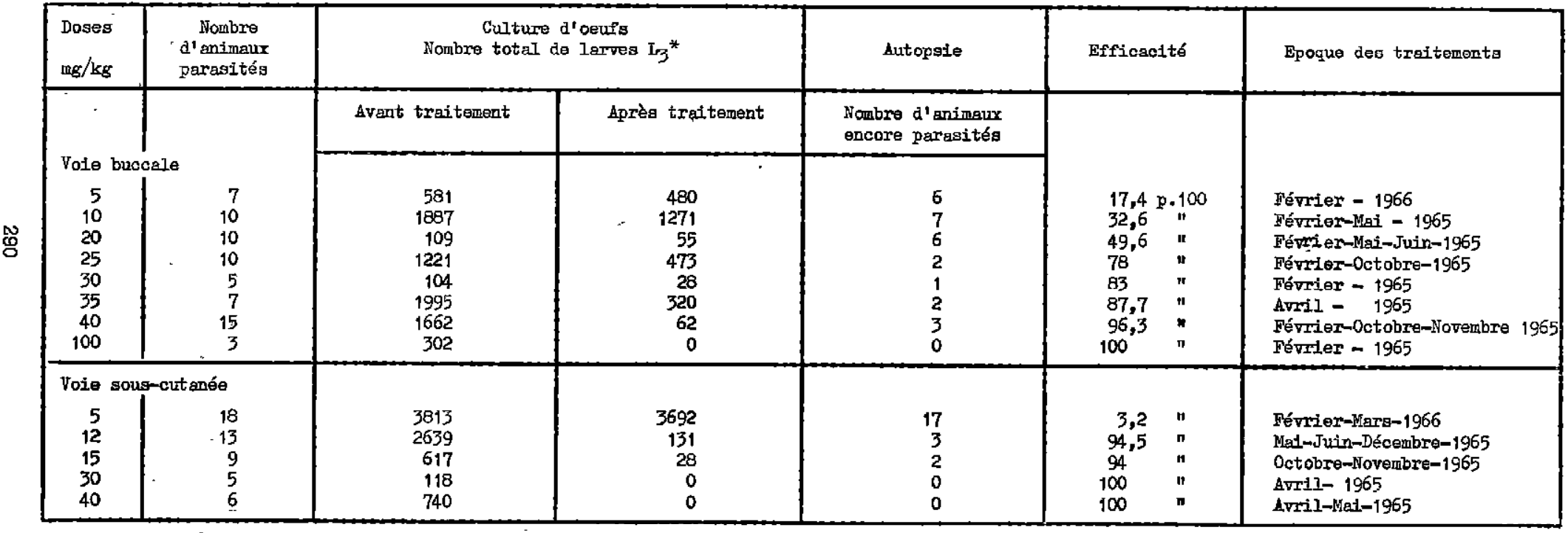

* Dane ume goitte de suspension aqueuse provenant dee bostes de Petri gyent servi aux coprocultures. 
TABLAU NOVI

sction du Tétramisole sur Gaigeria pachyacelis edulte.

\begin{tabular}{|c|c|c|c|c|c|}
\hline $\begin{array}{l}\text { Doses } \\
\text { mig/kg }\end{array}$ & $\begin{array}{l}\text { Nambre } \\
\text { d'animaux } \\
\text { paragités }\end{array}$ & $\begin{array}{l}\text { Nombre } \\
\text { de Nématodes } \\
\text { expulsés }\end{array}$ & $\begin{array}{l}\text { Frégence ou } \\
\text { absence }(-) \\
\text { des parasites } \\
\text { è l'autopsie }\end{array}$ & $\begin{array}{l}\text { Nombre } \\
\text { d'animaux } \\
\text { totalement } \\
\text { déparegités }\end{array}$ & Epoque des traitemento \\
\hline $\begin{array}{r}\text { Voie b } \\
10 \\
20 \\
25 \\
35 \\
40 \\
200\end{array}$ & $\begin{array}{l}3 \\
1 \\
5 \\
1 \\
2 \\
1\end{array}$ & $\begin{array}{r}53 \\
1 \\
16 \\
1 \\
7 \\
2\end{array}$ & $\begin{array}{l}- \\
- \\
- \\
-\end{array}$ & $\begin{array}{l}3 \text { sur } 3 \\
1 \\
5 \text { sur } 1 \\
5 \text { sur } 5 \\
1 \text { sur } 1 \\
2 \operatorname{sur} 2 \\
1 \text { sur } 1\end{array}$ & $\begin{array}{l}\text { Fétrier-Mai - } 1965 \\
\text { Février-Mai -Juin - } 1965 \\
\text { Février-Octobre - } 1965 \\
\text { Avril - 1965 } \\
\text { Février-0ctobre-Hovembre-1965 } \\
\text { Juin - } 1965\end{array}$ \\
\hline $\begin{array}{r}\text { Vole } \\
5 \\
12 \\
15 \\
30\end{array}$ & $\begin{array}{r}\text { utanée. } \\
1 \\
1 \\
1 \\
1\end{array}$ & $\begin{array}{l}2 \\
3 \\
2 \\
1\end{array}$ & $\begin{array}{l}- \\
- \\
-\end{array}$ & $\begin{array}{lll}1 & \text { gur } & 1 \\
1 & \text { sur } & 1 \\
1 & \text { aur } & 1 \\
1 & \text { sur } & 1\end{array}$ & $\begin{array}{l}\text { Férrier-Mai - } 1966 \\
\text { Mai-Juin-Décembre-1965 } \\
\text { Octobre-Novembre - } 1965 \\
\text { Arril } 1965\end{array}$ \\
\hline
\end{tabular}

TABLEAD WOVII

Action du Tétranieole sur Desophagostomum colvubianum adulto mûr.

\begin{tabular}{|c|c|c|c|c|c|}
\hline $\begin{array}{l}\text { Doses } \\
\text { ig/ty }\end{array}$ & $\begin{array}{l}\text { Nombre } \\
\text { d'animaux } \\
\text { parasités }\end{array}$ & $\begin{array}{c}\text { Nombre } \\
\text { d1 Oesophagostonum } \\
\text { expulséa }\end{array}$ & $\begin{array}{l}\text { Présence ou } \\
\text { absence (-) } \\
\text { de perasites } \\
\text { à l'autopsie }\end{array}$ & \begin{tabular}{l}
\multicolumn{1}{c}{ Nombre } \\
d'animaux \\
totalement \\
dépérasités \\
efficacité
\end{tabular} & Epoque des traitements \\
\hline \multicolumn{6}{|c|}{ Voie buccale. } \\
\hline 5 & 4 & 11 & 41 & 1 sur 4 & Février 1966 \\
\hline 10 & 5 & 234 & - & 5 sur 5 & Février-Maj-Jưn-1965 \\
\hline 20 & 6 & 104 & - & $\begin{array}{l}6 \text { sur } 6 \\
(100 p \cdot 100)\end{array}$ & Février-Octobre-1965 \\
\hline 25 & 5 & 49 & - & 5 our 5 & Février-Octobre-1965 \\
\hline 30 & 3 & 31 & - & $\begin{array}{l}3 \text { gur } 3 \\
(100 p \cdot 100)\end{array}$ & Février - 1965 \\
\hline 35 & 1 & 3 & - & 1 sur 1 & Mars-AqT11 - 1965 \\
\hline 40 & 3 & 38 & - & 3 sur 3 & Fétrier-0ctobre-Novembre-1965 \\
\hline 100 & 1 & 1 & - & 1 sur 1 & Fétrier - 1965 \\
\hline \multicolumn{6}{|c|}{ Voie sous-cut amée. } \\
\hline 5 & 15 & 307 & 83 & $\begin{array}{l}10 \text { sur } 15 \\
(21.5 p \cdot 100)\end{array}$ & Févrler-Hars-1966 \\
\hline 12 & 8 & 124 & - & $\begin{array}{l}8 \text { sur } 8 \\
(1000-100)\end{array}$ & Mei-Juin-Décembre-1965 \\
\hline 15 & 3 & 160 & - & $\begin{array}{l}3 \text { sur } 3 \\
(1000.100)\end{array}$ & Octobre-Norembre-1965 \\
\hline 30 & 2 & 21 & - & 2 sur 2 & dvril - 1965 \\
\hline 40 & 1 & 15 & - & 1 sur 1 & Avril - 1965 \\
\hline
\end{tabular}


TABLFAU WOVIII

Actín du Tétramlsole sur Haemoncus contortus.

\begin{tabular}{|c|c|c|c|c|c|c|}
\hline \multirow{2}{*}{$\begin{array}{l}\text { Doseg } \\
\mathrm{mg} / \mathrm{kg}\end{array}$} & \multirow{2}{*}{$\begin{array}{l}\text { Nombre } \\
\text { de moutions } \\
\text { utilisés }\end{array}$} & \multicolumn{2}{|c|}{$\begin{array}{l}\text { Culture d'oeufa } \\
\text { Nombre total de larras } \mathrm{I}_{3} \text { * }\end{array}$} & \multirow{2}{*}{$\begin{array}{l}\text { Absence de } \\
\text { parasites à } \\
\text { l'autopsie }\end{array}$} & \multirow{2}{*}{$\begin{array}{l}\text { Nombre de moutona } \\
\text { completement } \\
\text { déparasités } \\
\text { Efflcacité }\end{array}$} & \multirow{2}{*}{ Epoque des traitemente } \\
\hline & & Avant traitement & Après traitement & & & \\
\hline $\begin{array}{r}\text { Voie } \\
5 \\
10 \\
20 \\
25 \\
30 \\
35 \\
40\end{array}$ & $\begin{array}{l}5 \\
5 \\
5 \\
6 \\
1 \\
2 \\
4\end{array}$ & $\begin{array}{r}9 \\
8 \\
19 \\
24 \\
1 \\
2 \\
12\end{array}$ & $\begin{array}{l}0 \\
0 \\
0 \\
0 \\
0 \\
0 \\
0\end{array}$ & $\begin{array}{l}\bar{z} \\
\bar{z} \\
\bar{z}\end{array}$ & $\begin{array}{l}5 \text { sur } 5 \\
5 \text { our } 5 \\
5 \text { sur } 5 * * \\
6 \text { sur } 6 \\
1 \text { sur } 1 \\
2 \text { sur } 2 \\
4 \text { sur } 4\end{array}$ & $\begin{array}{l}\text { Février } 1966 \\
\text { Février-Maj-Juin-1965 } \\
\text { Février-Octobre-1965 } \\
\text { Février-Octobre-1965 } \\
\text { Février-1965 } \\
\text { Mars-Arril-1965 } \\
\text { Février-Octobre-Novembro } 1965\end{array}$ \\
\hline $\begin{array}{r}\text { Voie : } \\
5 \\
12 \\
15 \\
30 \\
40\end{array}$ & $\begin{array}{c}\text { cutanéo. } \\
11 \\
4 \\
5 \\
1 \\
4\end{array}$ & $\begin{array}{r}62 \\
8 \\
24 \\
1 \\
13\end{array}$ & $\begin{array}{l}0 \\
0 \\
0 \\
0 \\
0\end{array}$ & $\begin{array}{l}- \\
\overline{-} \\
\overline{-}\end{array}$ & $\begin{array}{l}11 \text { sur } 11 \\
4 \text { sur } 4 \\
5 \text { sur } 5 \\
1 \text { sur } 1 \\
4 \text { sur } 4\end{array}$ & $\begin{array}{l}\text { Févriler-Marg-1966 } \\
\text { Mai-Juin-Dócembre-1965 } \\
\text { Octobre-Notembre-1965 } \\
\text { Avril-1965 } \\
\text { Avril-1965 }\end{array}$ \\
\hline
\end{tabular}

***: forte infestation ;

* : feible infestetion;

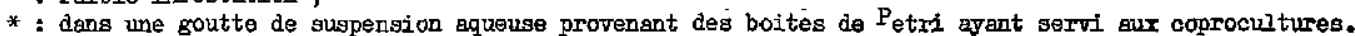


MABLELO HOIX

Action du Tétremisole our Buckleguris ovla et Buckleynuris globulosa.

\begin{tabular}{|c|c|c|c|c|c|}
\hline $\begin{array}{l}\text { Dosea } \\
\mathrm{mg} / \mathrm{kg}\end{array}$ & $\begin{array}{c}\text { Nombre } \\
\text { d'animaux } \\
\text { parasités }\end{array}$ & $\begin{array}{c}\text { Nombre } \\
\text { de Buakleyruria } \\
\text { expulsés }\end{array}$ & $\begin{array}{l}\text { Présence ou } \\
\text { absence (-) } \\
\text { de Nématodes } \\
\text { è } l^{\prime} \text { sutopale }\end{array}$ & $\begin{array}{l}\text { Nombre } \\
\text { d'animaux } \\
\text { totalement } \\
\text { déparasités }\end{array}$ & Epoque des traitements \\
\hline \multicolumn{6}{|c|}{ Voie buccale } \\
\hline $\begin{array}{r}20 \\
25 \\
30 \\
40 \\
100\end{array}$ & $\begin{array}{l}1 \\
1 \\
1 \\
1 \\
1\end{array}$ & $\begin{array}{l}1 \\
0 \\
1 \\
1 \\
1\end{array}$ & $\begin{array}{l}\overline{3} \\
\overline{-} \\
-\end{array}$ & $\begin{array}{lll}1 & \text { sur } 1 \\
0 & \text { sur } & 3 \\
1 & \text { sur } 1 \\
1 & \text { sur } 1 \\
1 & \text { sur } 1\end{array}$ & $\begin{array}{l}\text { Fétrier - } 1965 \\
\text { Octobre - } 1965 \\
\text { Février - } 1965 \\
\text { Octobre - } 1965 \\
\text { Février - } 1965\end{array}$ \\
\hline \multicolumn{6}{|c|}{ Voie sous-cutanée } \\
\hline $\begin{array}{r}5 \\
30\end{array}$ & $\begin{array}{l}3 \\
1\end{array}$ & $\begin{array}{l}1 \\
1\end{array}$ & 5 & $\begin{array}{l}1 \text { sur } 3 \\
1 \text { sur } 1\end{array}$ & $\begin{array}{l}\text { Février-Meri-1966 } \\
\text { Avril - } 1965\end{array}$ \\
\hline
\end{tabular}

TABLEATE NOX

sction dn Tétramisole sur les fornes arultes immatures d'Oesophagostomum columbianum

\begin{tabular}{|c|c|c|c|c|c|}
\hline $\begin{array}{l}\text { Doses } \\
\mathrm{mg} / \mathrm{kg}\end{array}$ & $\begin{array}{c}\text { Nombre } \\
\text { d'animalux } \\
\text { parasité }\end{array}$ & $\begin{array}{c}\text { Nombre } \\
\text { a'Oesophagostomum } \\
\text { expulsés }\end{array}$ & $\begin{array}{l}\text { Présence ou } \\
\text { absence ( } \rightarrow \text { ) } \\
\text { de parasitea } \\
\text { à l'autopsie }\end{array}$ & $\begin{array}{l}\text { Nombre } \\
\text { d'animauxx } \\
\text { totalement } \\
\text { déparasités } \\
\text { Efficacité }\end{array}$ & Epoque des treitements \\
\hline \multicolumn{6}{|c|}{ Vois buocale. } \\
\hline 10 & 4 & 19 & 31 & $1 \operatorname{sur} 4$ & Féviler-Ma1-1965 \\
\hline 20 & 2 & 11 & 12 & 1 gur 2 & Fétrier - 1965 \\
\hline 25 & 3 & 18 & - & 3 sur 3 & Février - 1965 \\
\hline 30 & 2 & 6 & - & 2 sur 2 & Févxier - 1965 \\
\hline 35 & 3 & 55 & - & 3 our 3 & Mars - Avril - 1965 \\
\hline 40 & 3 & 19 & - & 3 sur 3 & Février-Novembre-1965 \\
\hline 100 & 1 & 3 & - & 1 sur 1 & Février- 1965 \\
\hline \multicolumn{6}{|c|}{ Voie sous-cutianée. } \\
\hline $\begin{array}{l}12 \\
15 \\
30\end{array}$ & $\begin{array}{l}1 \\
1 \\
1\end{array}$ & $\begin{array}{l}1 \\
3 \\
4\end{array}$ & $\bar{z}$ & $\begin{array}{r}1 \text { sur } 1 \\
+1 \text { sur } 1 \\
1 \text { sur } 1\end{array}$ & $\begin{array}{l}\text { Mei - } 1965 \\
\text { Novembre }-1965 \\
\text { Atril - } 1965 \text {. }\end{array}$ \\
\hline
\end{tabular}

TABLEAD NOXI

Action du Tétramisole sur les formea Larvaires $L_{4}$ intranodulaites d'oesophagostomum columbianum.

\begin{tabular}{|c|c|c|c|c|}
\hline $\begin{array}{l}\text { Doges } \\
\mathrm{mg} / \mathrm{kg}\end{array}$ & $\begin{array}{c}\text { Nombre } \\
\text { d'ganimalx } \\
\text { paragités }\end{array}$ & $\begin{array}{l}\text { Nombre total de } \\
\text { nodures caecaur }\end{array}$ & $\begin{array}{l}\text { Nambre de } \mathrm{L}_{\text {f }} \\
\text { encore vivantes } \\
\text { a l'autopsie }\end{array}$ & Epoque des traitementr \\
\hline $\begin{array}{r}\text { Voie b } \\
5 \\
10 \\
20 \\
25 \\
30 \\
35 \\
40 \\
100\end{array}$ & $\begin{array}{r}3 \\
3 \\
1 \\
1 \\
2 \\
5 \\
13 \\
3\end{array}$ & $\begin{array}{r}18 \\
33 \\
1 \\
8 \\
15 \\
24 \\
76 \\
12\end{array}$ & $\begin{array}{r}3 \\
15 \\
1 \\
2 \\
4 \\
7 \\
26 \\
7\end{array}$ & $\begin{array}{l}\text { Février-Marg-1966 } \\
\text { Février-1965 } \\
\text { Février- } 1965 \\
\text { Février-0 ctobre-1965 } \\
\text { Février-1965 } \\
\text { Février-1965 } \\
\text { Pévrier-0ctobre-Hovembre-1965 } \\
\text { Pévrier-1965 }\end{array}$ \\
\hline $\begin{array}{r}\text { Voie s } \\
5 \\
12 \\
15 \\
30\end{array}$ & $\begin{array}{l}1 \\
1 \\
5 \\
1\end{array}$ & $\begin{array}{r}2 \\
5 \\
36 \\
5\end{array}$ & $\begin{array}{l} \\
1 \\
1 \\
9 \\
1\end{array}$ & $\begin{array}{l}\text { Février-Marz-1966 } \\
\text { Décembre-1965 } \\
\text { Octobre-Noverbre-1965 } \\
\text { Lvril-1965 }\end{array}$ \\
\hline
\end{tabular}


RABLEAU No

Action du Tétramisole sur les formes larvaires inmatures de Strongyloides papillosus

\begin{tabular}{|c|c|c|c|c|}
\hline $\begin{array}{l}\text { Doses } \\
\mathrm{mg} / \mathrm{kg}\end{array}$ & $\begin{array}{l}\text { Nombre de } \\
\text { moutons } \\
\text { utilisés }\end{array}$ & $\begin{array}{l}\text { lombre de moutons présentant } \\
\text { encore des formes imnatures } \\
\text { \& l'autopgie }\end{array}$ & $\begin{array}{l}\text { Nombre de } \\
\text { Pormes } \\
\text { immatures }\end{array}$ & Epoque des treitements \\
\hline $\begin{array}{r}\text { Voie b } \\
5 \\
25 \\
30 \\
35 \\
40 \\
100\end{array}$ & $\begin{array}{r}1 \\
10 \\
5 \\
7 \\
7 \\
3\end{array}$ & $\begin{array}{l}1 \\
1 \\
1 \\
2 \\
1 \\
0\end{array}$ & $\begin{array}{l}1 \\
5 \\
2 \\
2 \\
2 \\
0\end{array}$ & $\begin{array}{l}\text { Février - } 1966 \\
\text { Février-Octobre-1965 } \\
\text { Février - } 1965 \\
\text { Mars-Arril - } 1965 \\
\text { Pévrier-Octobre-Norembre-1965 } \\
\text { Février - } 1965\end{array}$ \\
\hline $\begin{array}{c}\text { Voie s } \\
12\end{array}$ & anée. & 1 & 1 & Mni $-J_{u L I n}-1965$ \\
\hline
\end{tabular}

sur Gaigeria pachyscelis. Buckleyuris ovis et Buckleyuris globulosa. Il est beaucoup moins valable. lorsqu'il s'agit de Strongyloides papillosus. Le pouvoir anthelminthique des deux médicaments à l'égard d'Haemoncus contortus, d'Oesophagostomum columbianum mûrs adulies ou immatures et d'Oesophagostomum columbianum à l'état de larves $L_{4}$ intranodulaires est du même ordre de grandeur.

Dans la lutte contre les Nématodes sévissant en milieu tropical, on ne peut que recommander les doses de $12 \mathrm{mg} / \mathrm{kg}$ par la voie sous-cutanée ou de $40 \mathrm{mg} / \mathrm{kg}$ par la voie buccale, car elles sont susceptibles de chasser dans la proportion de 90 p. 100 les principaux Nématodes (Strongyloides. Oesophagostomum. Gaigeria. Haemoncus et Buckleryuris) entrant dans la composition des associations parasitaires si fréquentes au Tchad et en $R, C, A$,

Par contre en Europe ou Haemoncus contortus est abondant, la dose de $5 \mathrm{mg} / \mathrm{kg}$ pourrait suffire lors d'infestations de faible ou de moyenne importance. Ces conclusions rejoignent, dans leurs grandes lignes, celles de THIENPONT et collaborateur (1966).

\section{III. - ACTIVITÉ DU MÉDICAMENT}

L'évacuation des parasites, dans tous les cas, commence aussitôt après la fin du traitement, Du fait de l'action propre du 16.535 R. P. sur les fibres lisses de l'intestin. le contenu de celui-ci est très rapidement repoussé vers l'extérieur avec les Nématodes qu'il renferme. N'apparaissent cependant ni les Strongyloides, ni les Haemoncus à quelques exceptions près.
Les délais d'expulsion sont alors les suivants: 24 heures après le traitement : 77 p. 100 des Nématodes éliminés.

48 heures après le traitement : 19 p. 100 des Nématodes éliminés.

96 heures après le traitement : 4 p. 100 des Nématodes éliminés.

Dans 96 p. 100 des cas, 50 heures environ après l'administration de l'anthelminthique aux doses indiquées, aucun parasite n'est plus visible dans les crottes et il n'existe plus de possibilité d'éclosion de larves infestantes $L_{3}$ de Gaigeria, d'Haemoncus et d'Oesophagosfomum columbianum.

A $40 \mathrm{mg} / \mathrm{kg}$ (V. B.) ef à $15 \mathrm{mg} / \mathrm{kg}$ (V.S. C.). les aufs de Strongyloides papillosus demeurent assez longtemps dans les selles : leur disparition totale demande 5 à 6 jours pendant lesquels le mouton reste dangereux et susceptible d'ensemencer le pâturage neuf sur lequel il est placé. II en est de même, lorsque le Tétramisole est utilisé d̀ $5 \mathrm{mg} / \mathrm{kg}$ contre Haemoncus contortus,

Le $16.535, R$. P. est donc le type parfait du nématodifuge.

\section{IV. - CONSÉQUENCES DU TRAITEMENT SUR LA SANTÉ DE L'ANIMAL.}

\section{A. - Conséquences visibles.}

Le médicament aux doses thérapeutiques est assez bien toléré. Seuls sont décelables pendant quelques minutes, surtout d̀ $40 \mathrm{mg} / \mathrm{kg}$ (voie buccale), des phénomènes d'excitation passagère avec accélération de la respiration, larmoiement, hypersalivation ef épreintes. Ces signes s'estompent rapidement. L'appétit est conservé, 


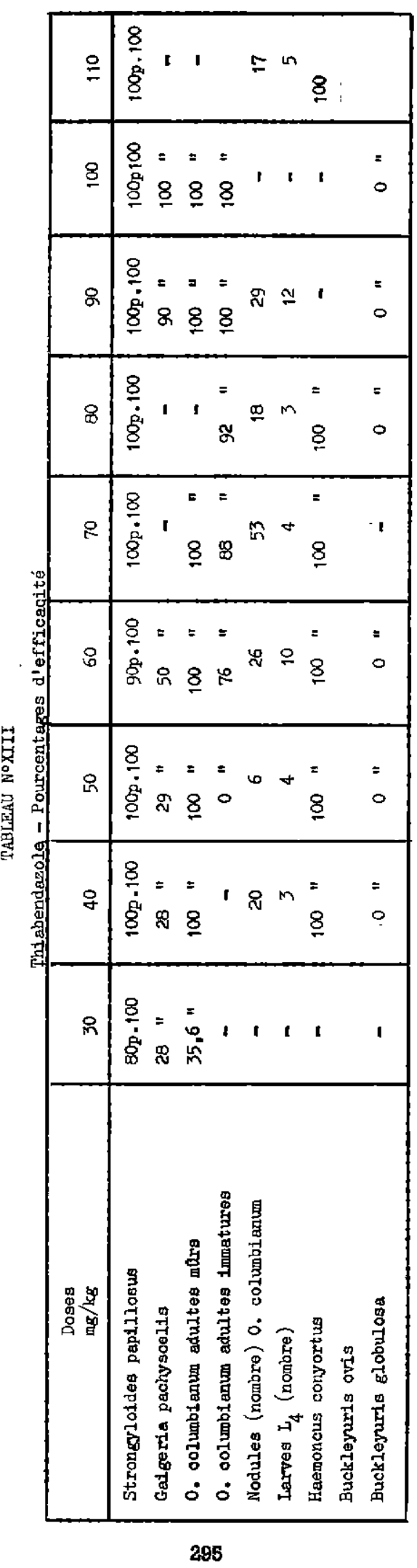


Grophique $N^{\circ} \mathrm{Il}$ Action du 16.535 RP sur Strongyloïdes

\section{Voie buccale}

______ Voie sous cutanée

Thiabendazole

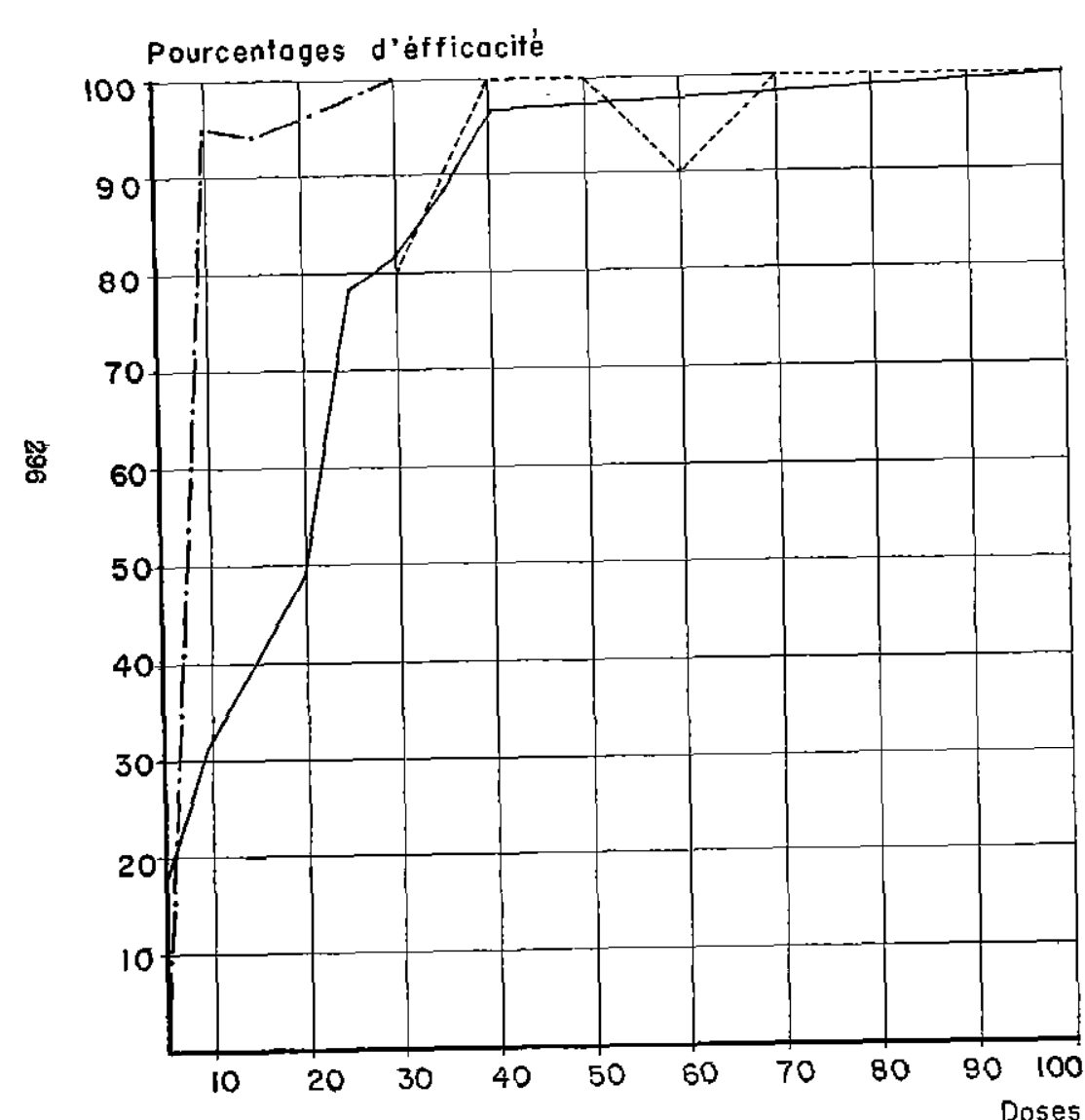

Pourcentages d'éfficacité

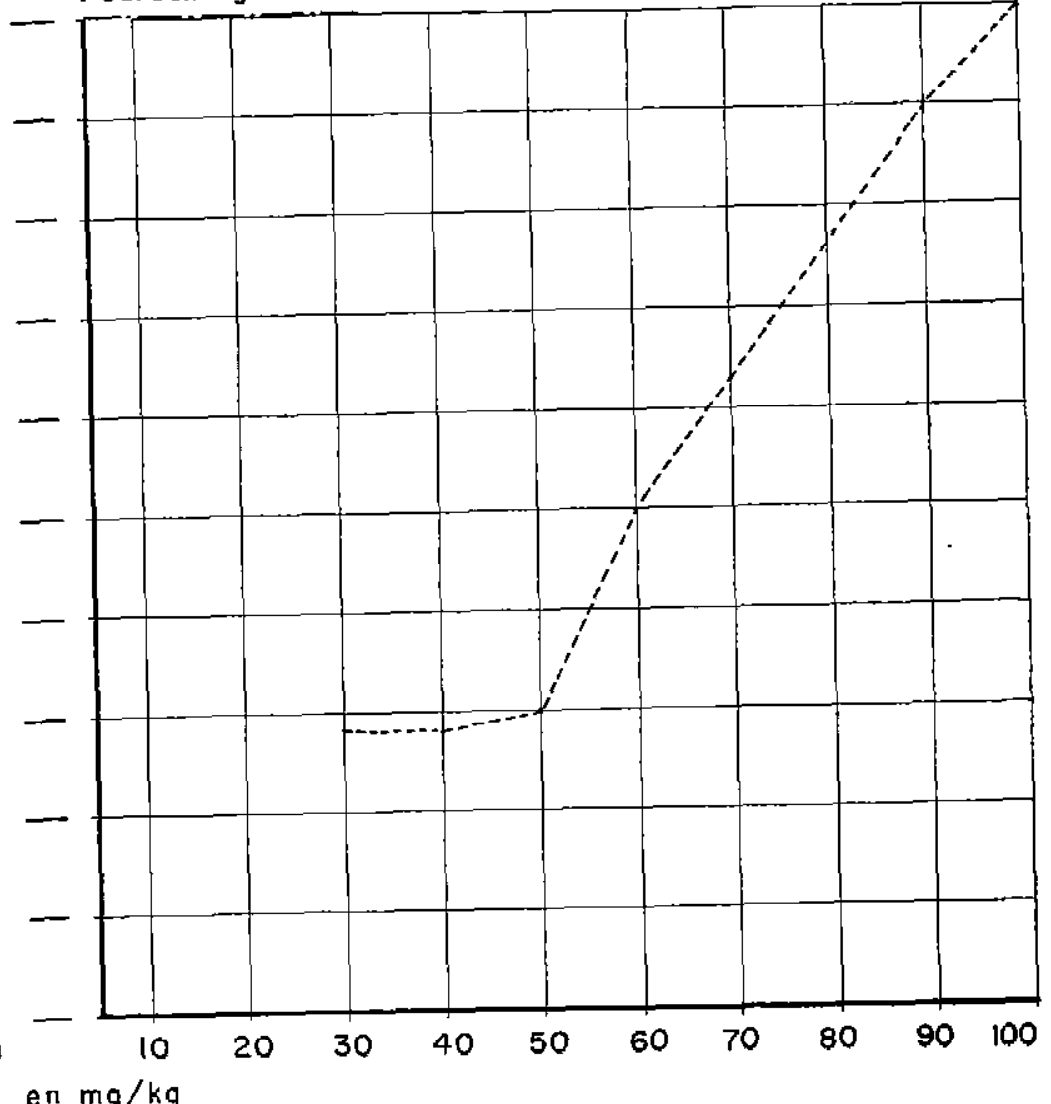


Graphique $N^{\circ}$ IV Action du 16.535 RP sur O. columbianum Adulte mur

Graphique $N^{\circ} \mathrm{V}$ Action du $16.535 \mathrm{RP}$ sur $O$. columbianum Aculte innature

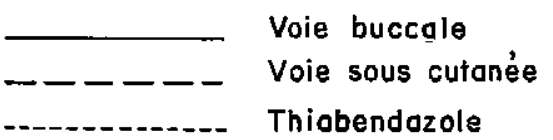

Pourcentoges d'èfficocité

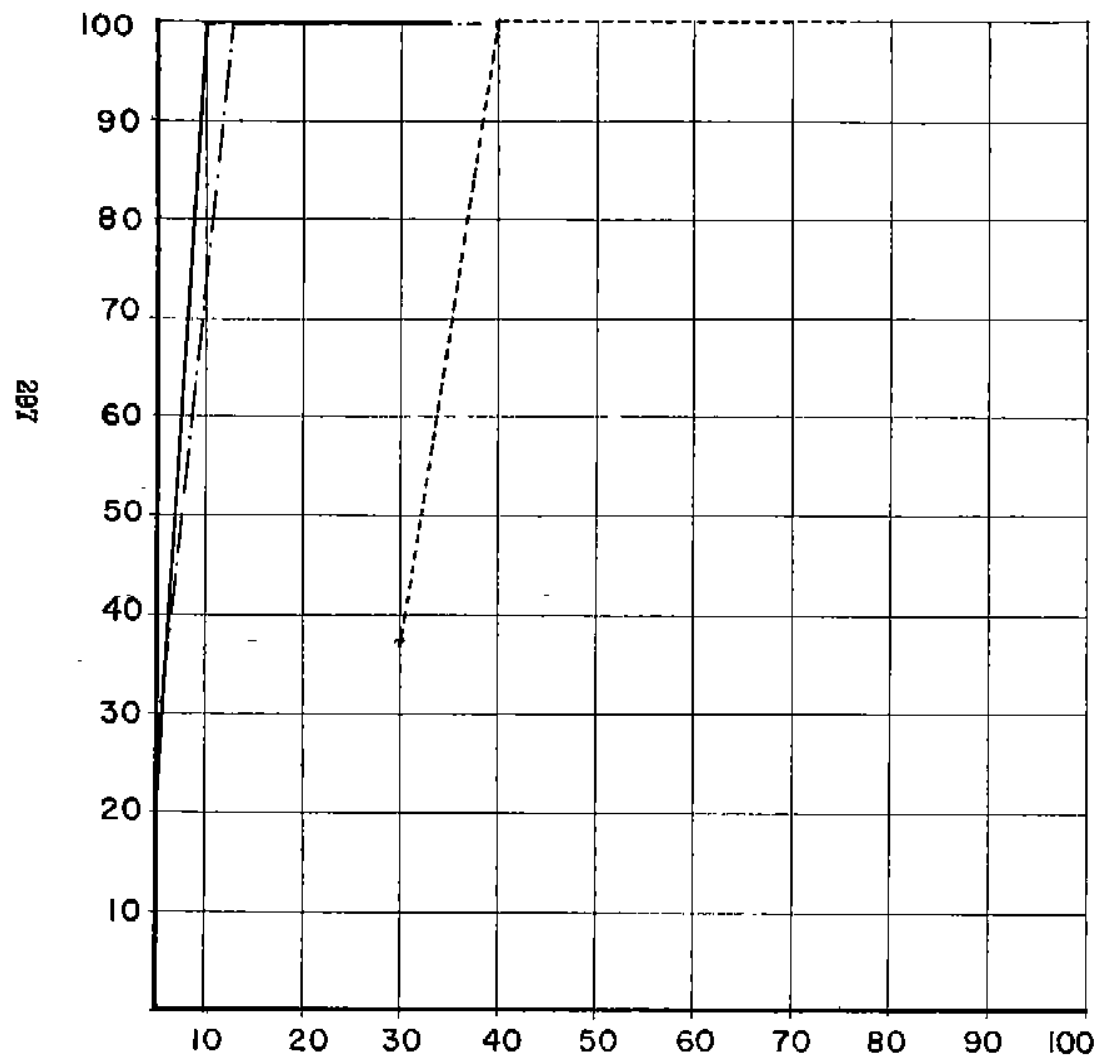

Doses en $\mathrm{mg} / \mathrm{kg}$
Pourcentages d'éfficacité

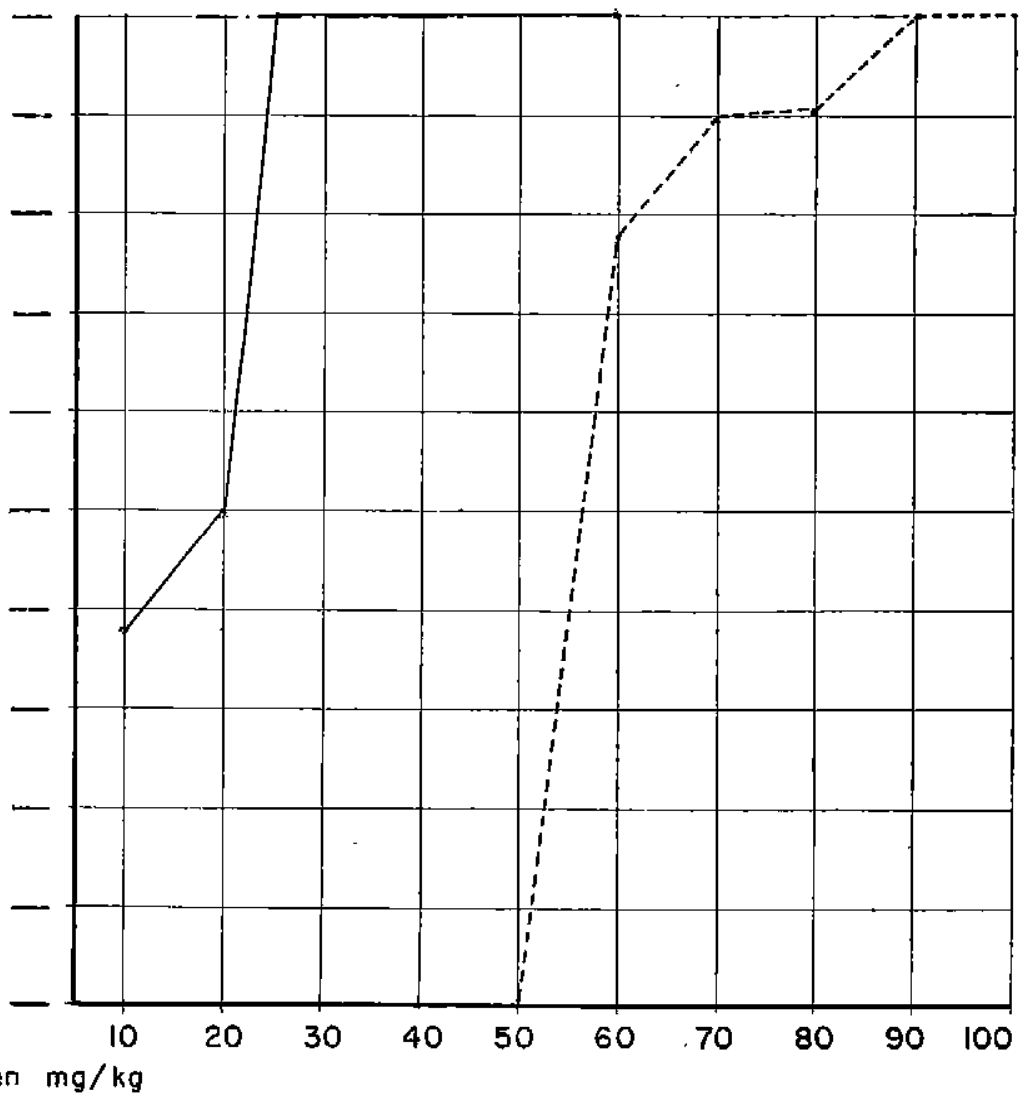


Graphique $N^{\circ}$ VI Relevès Climatiques

Température en $0^{\circ} \mathrm{C}$.

Degrè Hygrométrique

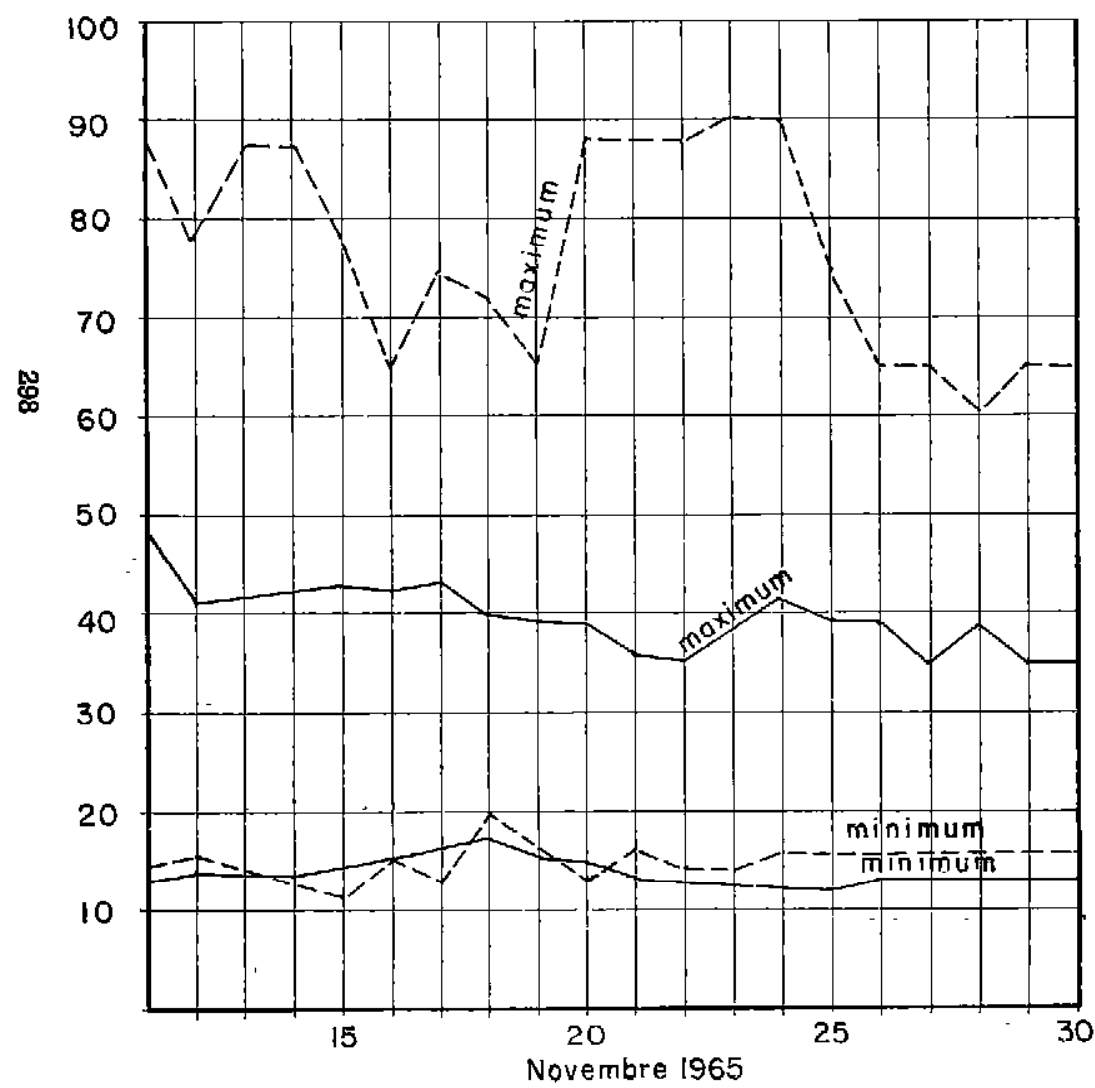

Grophique No VII Tetromisole Goins de Poids

Temoins

$16.535 \mathrm{RP} 15 \mathrm{mg} / \mathrm{kg}$ (V.S.C.)

$16.535 \mathrm{RP} 40 \mathrm{mg} / \mathrm{kg}$ ( V.B.)

$16.535 \mathrm{RP} 40 \mathrm{mg} / \mathrm{kg}+$ Yomesan $150 \mathrm{mg} / \mathrm{kg}$

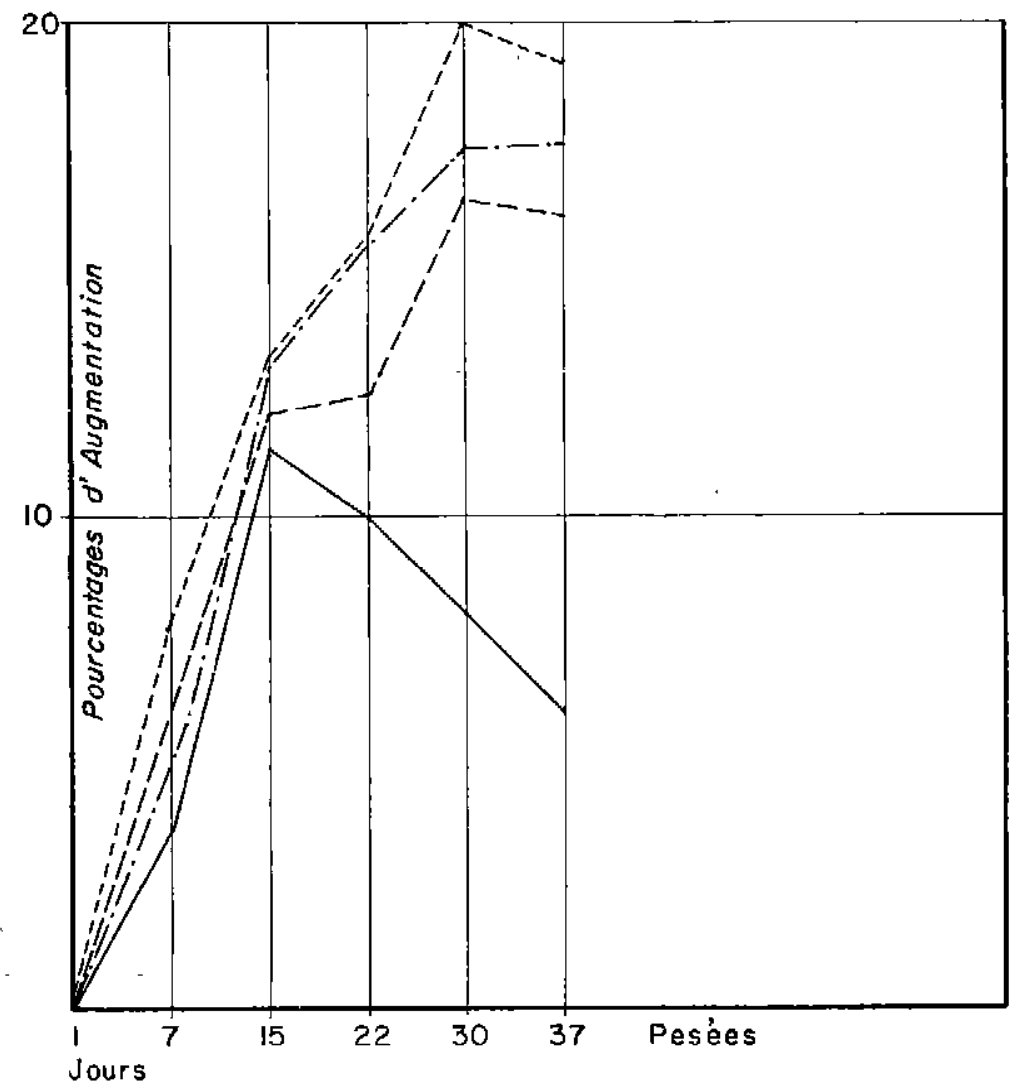


TASLBHA NOXIV

Protéines du sang; $15 \mathrm{mg} / \mathrm{kg}$ (V.S.C.)

\begin{tabular}{|c|c|c|c|c|c|c|}
\hline Noutons $\mathbb{I}^{\circ}$ & 15 & $\uparrow 7$ & 18 & 19 & 21 & l'oyenne \\
\hline $\begin{array}{l}\text { Protéines totiales } \\
\text { Avant traitement } \\
\text { Après traiteruent* }\end{array}$ & $\begin{array}{l}51,6 \\
60,3\end{array}$ & $\begin{array}{l}57 \\
63,7\end{array}$ & $\begin{array}{l}53,6 \\
60,3\end{array}$ & $\begin{array}{l}59,8 \\
63,8\end{array}$ & $\begin{array}{l}60,3 \\
67,7\end{array}$ & $\begin{array}{l}56,46 \\
63,16\end{array}$ \\
\hline $\begin{array}{l}\text { Globulinea } \\
\text { Avant traitement } \\
\text { Après traiteaent }\end{array}$ & $\begin{array}{l}27,9 \\
27,1\end{array}$ & $\begin{array}{l}28 \\
26,5\end{array}$ & $\begin{array}{l}27,9 \\
26,6\end{array}$ & $\begin{array}{l}28,4 \\
23,3\end{array}$ & $\begin{array}{l}29,8 \\
27,2\end{array}$ & $\begin{array}{r}28,40 \\
+\quad 26,12\end{array}$ \\
\hline $\begin{array}{l}\text { Albumines } \\
\text { Avant traitement } \\
\text { Après traitement }\end{array}$ & $\begin{array}{l}23,7 \\
33,2\end{array}$ & $\begin{array}{l}29 \\
37,2\end{array}$ & $\begin{array}{l}25,7 \\
33,7\end{array}$ & $\begin{array}{l}31,4 \\
40,5\end{array}$ & $\begin{array}{l}30,5 \\
40,5\end{array}$ & $\begin{array}{l}28,06 \\
37,04\end{array}$ \\
\hline $\begin{array}{l}\text { Poids } \\
\text { Avant traiteuert } \\
\text { Après traitemenl. }\end{array}$ & $\begin{array}{l}22 \\
22,3\end{array}$ & $\begin{array}{l}24 \\
26,2\end{array}$ & $\begin{array}{l}23 \\
25,5\end{array}$ & $\begin{array}{l}26 \\
29,7\end{array}$ & $\begin{array}{l}25 \\
28,2\end{array}$ & \\
\hline
\end{tabular}

* une semalre après atadministration du Tétramisole.

B. - Numérations globulaires-formules leucocytaires.

A $15 \mathrm{mg} / \mathrm{kg}$ (voie sous-cutanée), des sondages ont été effectués sur 5 moutons le premier jour du traitement ef le dernier jour, juste avant l'autopsie. On note :

- diminution du nombre d'hématies : 2 animaux.

- stabilité : 2 animaux.

- augmentation : 1 animal.

Les autres éléments de la formule sanguine ne subissent pas de modifications appréciables.

Les recherches * qui mettent en jeu la méthode de CORNVALL et Coli. ont porté sur deux séries d'ovins :

105 moutons reçoivent $15 \mathrm{mg} / \mathrm{kg}$ par la voie sous-cutanée en octobre 1965 (Tableau no XIV).

Les Globulines, dans l'ensemble, varient peu, avec cependant, dans certains cas, une plus forte valeur relative en début qu'en fin de traitement.

Seule, la Sérine croît de façon constante : l'augmentation est de 32,1 p. 100 en moyenne. II en résulte un accroissement des Protéines totales et du rapport Albumine/Globuline qui passe de 0,98 à 1,41 .

Le gain de poids est à mettre en parallèle avec l'augmentation des Protéines:

- Poids : 9,9 p. 100.

- Protéines : $+11,7$ p. 100.

* Aver lo collaboration de M. QUEVAL ef de Mme BRUNET,
20 4 animaux sont traités à la dose de $40 \mathrm{mg} / \mathrm{kg}$ par la voie buccale en novembre 1965 (Tableau $n^{\circ} \times \mathrm{V}$ ).

Sur ces animaux qui, outre les parasites habituels (Strongyloides, Gaigeria, Oesophogostomum et Haemoncus), hébergent des Oesophagostomes à l'état de larves $L_{4}$ et des Schistosomes, il y a encore accroissement des Protéines totales au profit des albumines ( $+7,3$ p. 100).

Ces deux essais, exécutés dans des conditions différentes. montrent que le Tétramisole, après destruction des Nématodes, a une action heureuse sur les Protéines du sang.

\section{V. - VALEUR ÉCONOMIQUE DU MÉDICAMENT}

Elle s'apprécie en fonction des gains de poids mesurés à la bascule.

Deux séries d'observation ont été effectuées :

10 En étable sur une période d'une semaine.

Ont été utilisés des lots d'animaux diversement parasités par Strongyloides, Goigeria. Haemoncus. Buckleyuris et Oesophagostomum adultes mûrs ou immatures.

Les moutons ont été nourris avec du foin composé en majeure partie de Brachiario. d'Echinochloo ef de Panicum. Des analyses faites à II. E. M. V. T. révèlent que, de sèptembre à décembre, ce foin a une valeur nutritive suffisante, valeur qui baisse sensiblement, au fur et à mesure que la saison sèche s'avance.

Les moutons ont été mis au repos 72 heures 
avant la première pesée et bien alimentés, de manière à supprimer tout risque d'erreur sur des animaux affamés quand ils parviennent au Laboratoire.

Les résultats sont les suivants: $(t a b, X V I)$.

Plusieurs remarques s'imposent :

a) En fin de saison sèche (juin), l'augmentation de poids est moins forte qu'en octobrenovembre ou qu'en janvier-février : c'est ce qui se passe notamment pour les doses de $12 \mathrm{mg} / \mathrm{kg}$ (juin $1965:+2$ p. 100) et de $15 \mathrm{mg} / \mathrm{kg}$ (octobre $1965:+9.9 \mathrm{p}, 100$ ) administrées par la voie souscutanée. Il s'agit d'une question d'alimentation : le déparasitage des animaux a des effets d'autant plus remarquables que la nourriture présentée par la suite est plus abondante et plus riche. Or, en mai-juin. les possibilités fourragères du Tchad sont faibles et les herbes sèches et peu nutritives.

b) Aux doses de 5, 10,20 et $25 \mathrm{mg} / \mathrm{kg}$ par la voie buccale, les différences enregistrées entre les lots proviennent du fait que les moutons traités en février $1965(10,20$ et $25 \mathrm{mg} / \mathrm{kg}$ ) étaient porteurs d'un grand nombre d'Anaplocephalidae (Stilesia globipunctata), qui, s'ils pullulent, peuvent nuire grandement à la santé de

TABLI,AU NOXV

Protéines du sang.

\begin{tabular}{|c|c|c|c|c|c|}
\hline Wioutong NC & 31 & $\$ 2$ & 33 & 34 & Woyenne \\
\hline $\begin{array}{l}\text { Protézres totales } \\
\text { Avant trìtement } \\
\text { Après traitement }\end{array}$ & $\begin{array}{l}37,50 \\
65,52\end{array}$ & $\begin{array}{l}56,16 \\
56,16\end{array}$ & $\begin{array}{l}64,00 \\
66,96\end{array}$ & $\begin{array}{l}61,92 \\
60,48\end{array}$ & $\begin{array}{l}59,94 \\
62,28\end{array}$ \\
\hline $\begin{array}{l}\text { Globulines } \\
\text { Avant triltement } \\
\text { Après traitemert }\end{array}$ & $\begin{array}{l}32,25 \\
30,96\end{array}$ & $\begin{array}{l}29,67 \\
30,96\end{array}$ & $\begin{array}{l}30,96 \\
30,96\end{array}$ & $\begin{array}{l}29,67 \\
30,44\end{array}$ & $\begin{array}{l}30,64 \\
30,84\end{array}$ \\
\hline $\begin{array}{l}\text { Albumines } \\
\text { Avent traitement } \\
\text { Après traitement }\end{array}$ & $\begin{array}{l}25,35 \\
34,56\end{array}$ & $\begin{array}{l}26,49 \\
25,20\end{array}$ & $\begin{array}{l}33,12 \\
56\end{array}$ & $\begin{array}{l}52,25 \\
30,03\end{array}$ & $\begin{array}{l}29,30 \\
31,45\end{array}$ \\
\hline $\begin{array}{l}\text { Pouds } \\
\text { Avant traat ement } \\
\text { Après traitement }\end{array}$ & $\begin{array}{l}31 \\
32\end{array}$ & $\begin{array}{l}30 \\
35\end{array}$ & $\begin{array}{l}30 \\
35\end{array}$ & $\begin{array}{l}31 \\
35\end{array}$ & $2,4 \mathrm{p} \cdot 100 *$ \\
\hline
\end{tabular}

* une semazre aprés l'aumaristration du Tétramisole.

TABLEAU NOXVI

Augmentation de poids en étable

\begin{tabular}{|c|c|c|c|c|c|c|c|}
\hline \multicolumn{8}{|c|}{ Vole buccale } \\
\hline $\begin{array}{l}\text { Doseg } \\
\mathrm{mg} / \mathrm{kg}\end{array}$ & 5 & 10 & .20 & 25 & 30 & 40 & 100 \\
\hline $\begin{array}{l}\text { Poids total des animaux* } \\
\text { Avant traitement } \\
\text { Après traltement }\end{array}$ & $\begin{array}{l}220 \\
238\end{array}$ & $\begin{array}{l}300 \\
306\end{array}$ & $\begin{array}{l}213 \\
224\end{array}$ & $\begin{array}{l}238 \\
256,5\end{array}$ & $\begin{array}{l}105 \\
115 \\
\end{array}$ & $\begin{array}{l}103 \\
113\end{array}$ & $\begin{array}{l}81 \\
90\end{array}$ \\
\hline Nombse $d^{3}$ animaux & 7 & 9 & 7 & 9 & 4 & 4 & 3 \\
\hline Pourcentage d'eugmentation & $+8 p \cdot 100$ & $+2 \mathrm{p} \cdot 100$ & $+5,1 p 100$ & $+7,7 \mathrm{p} 100$ & $+9,5,100$ & $+9,7 p 100$ & $+11 \mathrm{p} \cdot 100$ \\
\hline \multicolumn{8}{|c|}{ Voie sous-cutanée } \\
\hline $\begin{array}{l}\text { Doses } \\
\mathrm{mg} / \mathrm{kg}\end{array}$ & & 5 & & 12 & $i$ & & 15 \\
\hline $\begin{array}{l}\text { Poids total 'des animaux*. } \\
\text { Avant traitement } \\
\text { Après' traitement }\end{array}$ & \multicolumn{2}{|c|}{$\begin{array}{l}521 \\
571\end{array}$} & \multicolumn{3}{|c|}{$\begin{array}{l}240 \\
245 \\
\end{array}$} & \multicolumn{2}{|c|}{$\begin{array}{l}120 \\
131,9\end{array}$} \\
\hline Nombre d'animaur & \multicolumn{2}{|c|}{17} & \multicolumn{3}{|c|}{10} & \multicolumn{2}{|r|}{5} \\
\hline Pourcentage d'augmentation & \multicolumn{2}{|c|}{$+9,5$ p. 100} & \multicolumn{3}{|c|}{$+2 p \cdot 100$} & \multicolumn{2}{|c|}{$+9,9 p \cdot 100$} \\
\hline
\end{tabular}

* en kilogrammes 
I'animal. Par contre. en février $1966(5 \mathrm{mg} / \mathrm{kg})$. les Cestodes intestinaux étaient, dans l'ensemble beaucoup plus rares.

c) La présence de Schistosoma bovis dans les veines mésentériques et de larves $L_{4}$ intranodulaires d'Oesophagosiomum columbionum. en plus des Nématodes adultes les plus communs, fausse également les résultats: à $40 \mathrm{mg} / \mathrm{kg}\left(V . B_{\text {. }}\right.$ ), le gain de poids n'est plus que de 2.4 p. 100 , ainsi qu'il a été dit plus haut.

Il n'en demeure pas moins qu'après le traitement au Tétramisale - si le fourrage est de bonne qualité - les prises de poids sont rapides. L'appétit s'accroît, la consommation de foin s'élève' dans des proportions notables ef les animaux maigres se rétablissent rapidement,

20 Sur le terrain (six semaines),

Les essais se sont déroulés du 11-11-1965 au 18-12-1965. Pour cette expérience. 20 animaux fortement parasités ont été choisis :

- cing d'entre eux ont servi de témoins (lot A),

- cinq cutres oni reçu $15 \mathrm{mg} / \mathrm{kg}(\mathrm{V}, \mathrm{S}, \mathrm{C}$, ) lot $B$ ),

of $C$ ),

- les cing derniers ont reçu $40 \mathrm{mg} / \mathrm{kg}$ de Tétramisole $\left(\mathrm{V}, \mathrm{B}_{1}\right.$ ) et $150 \mathrm{mg} / \mathrm{kg}$ de YOMESAN BAYER, (lot D).

Aussitôt après le traitement. les moutons ont été placés sur un pâturage de saison sèche peu dense. composé de graminées diverses d̀ l'état de paille et d'arbustes épineux dont les moutons sont friands.

Le graphique $n^{\circ} \mathrm{Vl}$ indique les conditions de température et-d'hygrométrie régnant à cette époque sur la zone considérée, voisine du Laboratoire de FARCHA $(10 \mathrm{~km})$.

Les animaux ont été pesés régulièrement cha- que semaine durant 5 semaines (Tableau $n^{\circ} \mathrm{XVII}$ ).

Les résultats s'avèrent excellents : outre la disparition quasi totale des Nématodes, à l'autopsie, le traitement au Tétramisole aux doses de $15 \mathrm{mg} / \mathrm{kg}$ (V. S. C.) et de $40 \mathrm{mg} / \mathrm{kg}$ (V. B.) se traduit, par rapport aux témoins par un gain de poids qui oscille autour de 10,9-13,7 p. 100 (graph. VIl). Dans les conditions de cette expérience. l'adjonction d'un Taenicide (YOMESAN BAYER) n'offre guère d'intérêt. les prises de poids dans le lot $D$ n'étant pas supérieures à ce qu'elles sont avec le Tétramisole seul.

La valeur économique du 16,535 R. P. est donc indéniable, car ce médicament. tout en faisant disparaître les Nématodes du tube digestif, est susceptible - si les conditions alimentaires sont convenables - d'élever sensiblement la productivité du troupeau ovin.

\section{VI. - TOXICITÉ}

\section{Toxicité pour les moutons adultes.}

Des doses progressivement croissantes ont été expérimentées : (tab. XVIII).

Le 16.535 R. P., dans les conditions tchadiennes, tue la totalité des animaux traités:

- par la voie buccale. entre 180 et $200 \mathrm{mg} / \mathrm{kg}$, ce qui donne un coefficient chimiothérapique compris entre 4,5 et 5 , si l'on tient compte de la dose thérapeutique de $40 \mathrm{mg} / \mathrm{kg}$,

- par la voies ous-cutanée, entre 60 et $65 \mathrm{mg} / \mathrm{kg}$ : le coefficient chimiothérapique oscille alors autour de 5-5,4i en partant de la dose de $12 \mathrm{mg} / \mathrm{kg}$.

Chez le mouton, le 16,535 R. P. semble donc mieux toléré par la voie sous-cutanée que par. la voie buccale.

En outre, le Tétramisole par voie buccale est

TABLEAU NoXVII

Augmentation de poids sur le terrain

\begin{tabular}{|c|c|c|c|c|c|c|c|c|}
\hline \multirow{2}{*}{$\begin{array}{l}\text { Dates de } \\
\text { pegáe }\end{array}$} & \multicolumn{4}{|c|}{ Poids total (en kilogrammes) } & \multicolumn{4}{|c|}{ Augmentation de poids (en pourcentage) } \\
\hline & Lot $A$. & Lot $B$. & Lot C. & Lot $D$. & Lot $A$ & Lot $B$. & Lot C. & Lot $D$. \\
\hline $\begin{array}{r}11-11-65 \\
19-11-65 \\
27-11-65 \\
4-12-65 \\
11-12-65 \\
18-12-65\end{array}$ & $\begin{array}{l}153 \\
159 \\
170 \\
169 \\
165 \\
151\end{array}$ & $\begin{array}{l}155 \\
164 \\
173 \\
174 \\
181 \\
180\end{array}$ & $\begin{array}{l}137 \\
147 \\
154 \\
158 \\
164 \\
163\end{array}$ & $\begin{array}{l}154 \\
163 \\
173 \\
176 \\
181 \\
101\end{array}$ & $\begin{array}{l}=\overline{-} \\
+3,9 p \cdot 100 \\
+11,1 \\
+10 " \\
+7,7 " \\
+5,2 "\end{array}$ & $\begin{array}{l}- \\
+5,8 p \cdot 100 \\
+11,6 \pi \\
+12,3 \pi \\
+16,7 \pi \\
+16,1 \pi\end{array}$ & $\begin{array}{l} \\
+7, \overline{2} \cdot 100 \\
+12,4 \quad " \\
+15,3 \quad n \\
+19,7 \quad n \\
+18,9 \quad n\end{array}$ & $\begin{array}{l}-5 \\
+5,8 \mathrm{p} \cdot 100 \\
+12,4 \quad " 1 \\
+15,3 \quad 11 \\
+17,5 \quad 7 \\
+17,5 \quad 11\end{array}$ \\
\hline
\end{tabular}


TABLEAT NOXVIIT

Mortalcté des moutons fidultes

\begin{tabular}{|c|c|c|c|}
\hline $\begin{array}{l}\text { Dosess } \\
\mathrm{mg} / \mathrm{kg}\end{array}$ & $\begin{array}{c}\text { Nombre de moutons } \\
\text { utilisés }\end{array}$ & Mortallté & Epoque des traitementa \\
\hline $\begin{array}{l}\text { Voie buccale } \\
5 \\
10 \\
20 \\
25 \\
30 \\
35 \\
40 \\
100 \\
150 \\
130 \\
200 \\
225 \\
250\end{array}$ & $\begin{array}{r}7 \\
10 \\
10 \\
10 \\
5 \\
7 \\
16 \\
3 \\
3 \\
2 \\
3 \\
2 \\
4\end{array}$ & $\begin{array}{l}0 . \\
0 \\
0 \\
0 \\
0 \\
0 \\
0 \\
0 \\
1 \\
1 \\
3 \\
2 \\
4\end{array}$ & $\begin{array}{l}\quad \text { ' } \\
\text { Février-Narg } 1966 \\
\text { Février-Juin } 1965 \\
\text { Février-Juin 1965 } \\
\text { Février-Octobre } 1965 . \\
\text { Février } 1965 \\
\text { Arrtl 1965 } \\
\text { Février-Octobre-Novembro } 1965 \\
\text { Février } 1965 \\
\text { Juin } 1965 \\
\text { Mars } 1966 \\
\text { Février } 1965 \\
\text { Mars } 1965 \\
\text { Mars } 1965\end{array}$ \\
\hline $\begin{array}{l}\text { Voie sous-cut } \\
5 \\
10 \\
15 \\
30 \\
40 \\
50 \\
60 \\
65 \\
70\end{array}$ & $\begin{array}{r}18 \\
18 \\
11 \\
6 \\
6 \\
6 \\
13 \\
2 \\
3\end{array}$ & $\begin{array}{l}0 \\
0 \\
0 \\
0 \\
0 \\
2 \\
1 \\
2 \\
3\end{array}$ & $\begin{array}{l}\text { Févrtier-Mare } 1966 \\
\text { Juin-Novembre-Décembre } 1965 \\
\text { Oetobre-Novembre-Décembre } 1965 \\
\text { Arril } 1965 \\
\text { Avril } 1965 \\
\text { Juin } 1965 \\
\text { Juin } 1965 \\
\text { Mars } 1966 \\
\text { Juin } 1965\end{array}$ \\
\hline
\end{tabular}

environ deux fois plus toxique que le Thiabendazole administré de la même façon.

a) Symptômes de l'intoxication au Tétramisole,

Quelques minutes après le traitement, on observe :

- de l'inquiétude,

"- de l'excitation : le mouton cherche à fuir, en brisant tous les obstacles placés sur son passage.

Cette phase d'excitation est suivie d'une phase de viólente agitation : mouvements en rond à grande vitesse. bonds désordonnés. L'animal est couvert de sueur.

De très fortes coliques apparaissent bientôt : le 16.535 R. P. agit sur les fibres lisses de l'intestin. avec défécations nombreuses et répétées souvent accompagnées d'efforts douloureux. Le mouton se couche et se relève sans cesse. La respiration s'accélère et le cœur se met à battre d̀ un rythme anormal. En même temps. le médicament agit sur les glandes lacrymales (larmoiement) et sur les glandes salivaires (abondante salivation et mousse blanchâtre au coin des lèvres).

Au bout de 10 minutes. les signes d'intoxica- tion s'amplifient : le 16,535 R. P. atteint les centres nerveux supérieurs. On note du tournoiement. des mouvements de mastication et des grincements de dents. Les yeux sont révulsés. L'animal semble « fou 》. 11 tombe :

, - soit sur place. d'un seul coup, les quatre pattes écartées et meurt.

- soit en, décubitus latéral, tête en extension. Les membres sont agités de mouvements violents et les muscles superficiels en état de fibrillation permanente. Dans ce cas. l'animal met au total un quart d'heure, pour mourir.

L'intoxication par le Tétramisole esf' extrêmement spectaculaire.

Cette description est valable que le médicament soit administré par la voie buccale ou par la voie sous-cutanée.

\section{b) Lésions.}

A l'œil nu. elles sont fort discrètes et peu marquées. On relève :

- une forte congestion intestinale sans hémorragies.

- de la congestion rénale.

- un œdème du poumon très prononcé. 
- la congestion cérébrale, surtout au niveau du cervelet et du bulbe. est peu. visible.

Lorsque la mort survient en 5 jours - comme c'est le cas à $200 \mathrm{mg} / \mathrm{kg}$ - le foie prend une teinte feuille morte.

\section{$2^{\circ}$ Toxicité pour les femelles gestanies.}

9 brebis gestantes ont été traitées le 19-11-65.

a) $15 \mathrm{mg} / \mathrm{kg}$ sous la peau (Tab. $X \mid X$ ).

b) $40 \mathrm{mg} / \mathrm{kg}$ par la voie buccale (Tab. $X X$ ).

Le traitement au Tétramisole ne parâ̂t pas apporter de modifications sensibles à l'état des femelies gestantes. Aucun avortement n'a été relaté ef les jeunes étaient normaux à la naissance.

Du point de vue production de lait, la croissance des jeunes est normale : la sécrétion lactée n'a donc pas été gênée par des traitements effectués quelques jours avant la mise à bas.

\section{$3^{\circ}$ Toxicité pour les agneaux de lait.}

6 agneaux âgés de 1 mois-1 mois et demi ont reçu $40 \mathrm{mg} / \mathrm{kg}$ de Tétramisole (V. B.) le 13-11966 (Tab. XX1).

Le médicament ne semble pas avoir d'effets nocifs sur la santé des agneaux de lait. L'administration du Tétramisole au moment du sevrage permet aux jeunes de franchir sans encombre ce cap difficile et la courbe de croissance est alors très favorable.

\section{CONCLUSIONS}

Lors d'essais effectués en 1965-1966 sur 259 ovins originaireśs des régions Ouest $d u$ Tchad, il a été constaté :

A. - - Du point de vue pouvoir anthelminthique, le Tétramisole. sur des moutons n'ayant subi aucune préparation :

10 Est totalement inactif sur Schistosoma bovis, Paramphistomum microbothrium, Cormyerius popillatus, Moniezia expansa, Stilesia hepatica, Stilesia globipunctato, Avifellina centripunctoto et Avitellina woodlandi.

$2^{\circ}$ Gaigeria pachyscelis est détruit à partir de $10 \mathrm{mg} / \mathrm{kg}$ (V. B. *) ef de $5 \mathrm{mg} / \mathrm{kg}$ (V. S. C. **).

* V. B. = Voie buccale.

** V. S. C. $=$ Voie sous-cutanée. $3^{\circ}$ Les Haemoncus contortus de la caillette, en cas d'infestations moyennes, sont tués à partir de $5 \mathrm{mg} / \mathrm{kg}$, quel que soit le mode d'administration.

40 Les formes adultes mûres ou immatures d'Oesophagostomum columbianum sont éliminées vers $25 \mathrm{mg} / \mathrm{kg}$ (V. B.) et $12 \mathrm{mg} / \mathrm{kg}$ (V.S. C.). Les formes larvaires intranodulaires $L_{4}$ du même parasite survivent en grand nombre (24 à 33 p. 100), même si les doses sont élevées.

5095 p. 100 environ des formes adultes de Strongyloides papillosus disparaissent à $40 \mathrm{mg} / \mathrm{kg}$ (V.B.) ou à $12 \mathrm{mg} / \mathrm{kg}$ (V.S.C.). demeurent présentes également quelques formes larvarres immatures en très petit nombre.

60 L'action sur Buckleyuris ovis et sur Buckleyuris globuloso paraît plus irrégulière.

$7^{\circ}$ Le Tétramisole est donc supérieur au Thiabendazole $(100 \mathrm{mg} / \mathrm{kg}$ ) si l'on a affaire à Buckleyuris ou Gaigeria, égal lorsqu'il s'agit d'Hoemoncus ou d'Oesophagostomum. un peu inférieur pour Strongyloides papillosus.

8o En milieu tropical où les associations de Nématodes sont fréquentes deux dosages peuvent être recommandés :

- $40 \mathrm{mg} / \mathrm{kg}$ par la voie buccale.

- $12 \mathrm{mg} / \mathrm{kg}$ par la voie sous-cutanée, après préparation extemporanée d'une solution à 10 p. 100.

B. - Du point de vue mode d'action, le 16.535 R. P. se comporte comme un Nématodifuge. A $40 \mathrm{mg} / \mathrm{kg}$ (V. B.) et à $12 \mathrm{mg} / \mathrm{kg}$ (V. S. C.), les « gros Nématodes 》, dans 96 p. 100 des cas, cessent d'être visibles 50 heures après l'administration du médicament. A ces doses cependant, des cufs de Strongyloides persistent au moins pendant 6 jours, d'où possibilités de contamination ultérieure des pâturages neufs, si un certain délai d'isolement n'est pas observé après le traitement.

C. - Du point de vue voleur économique, en étable, si l'on est en présence d'un polyparasitisme uniquement à base de Nématodes, l'augmentation de poids en une semaine est de : $+9,7$ p. 100 à $40 \mathrm{mg} / \mathrm{kg}$ et de $+9,9$ p. 100 à $15 \mathrm{mg} / \mathrm{kg}$.

Sur le terrain. au bout de cinq semaines. le gain de polds est de $+10,9-13.7$ p. 100. Les 
TABLFAU NOXIX

\begin{tabular}{|c|c|c|c|c|}
\hline Brebis $\pi^{\circ}$ & \multicolumn{2}{|c|}{ Poids } & Niige bas & Aspect des jeumes \\
\hline 23 & $19-11-65$ & $25-11-65$ & & Normal \\
24 & 41 & 45 & $26-11-1965$ & Deux jeunes normaux \\
27 & 53 & 54 & $28-11-1965$ & mais peu de lait. \\
30 & 44 & 46 & Gestante de 2 & Pas d'avortement \\
& 40 & 41 & Nois. & Normai \\
\hline
\end{tabular}

TABLEAJ NOXX

\begin{tabular}{|c|c|c|c|c|}
\hline Brebia $n^{\circ}$ & \multicolumn{2}{|c|}{ Poids } & Mise bas & Aspect des jeunes \\
\hline 22 & $19-11-65$ & $25-11-65$ & & Normal \\
25 & 36 & 40 & $26-11-1965$ & $n$ \\
26 & 39 & 39 & $6-12-1965$ & $n$ \\
29 & 45 & 47 & $5-12-1965$ & " \\
28 & 35 & - & $23-11-1965$ & Pas d'avartement \\
\hline
\end{tabular}

TABLEAU NOXXI

\begin{tabular}{|c|c|c|c|c|}
\hline Agneaux $n^{\circ}$ & Date de naisoance & $\begin{array}{c}13-1-1966 \\
\text { (traitement) }\end{array}$ & $12-3-1966$ & Tolérance \\
\hline 1 & $23-1:-1965$ & 12 & 15 & excellente \\
\hline 2 & $6-12-1965$ & 5 & 9 : & $n$ \\
\hline 3 & $26-11-1965$ & 11 & 19 & $n$ \\
\hline 4 & $5-12-1965$ & 7 & 12 & $n$ \\
\hline 5 & $3-12-1965$ & 6 & 10 & $n$ \\
\hline 6 & $28-11-1965$ & $B$ & $\begin{array}{c}15,8 \\
+63,2 \mathrm{p} .100\end{array}$ & " \\
\hline
\end{tabular}

répercussions sur la santé de l'animal sont nulles. On note un fort accroissement des Protérnes du sang.

D. - Du point de vue toxicité.

- Par la voie buccale (40 mg/kg), le coefficient chimiothérapique oscille autour de 4,5-5.

- par la voie sous-cutanée $(12 \mathrm{mg} / \mathrm{kg})$, il varie entre 5 et 5,4 .

Le 16.535 R. P. est donc un peu moins toxique par la vole sous-cutanée que par la voie buccale. II est en tous cas deux fois plus toxique que le Thiabendazole.

Vers $200-225 \mathrm{mg} / \mathrm{kg}(V$. B.) et $60-70 \mathrm{mg} / \mathrm{kg}$ (V. S. C.), les moutons meurent en 20 minutes environ avec, dans un premier temps, des signes d'excitation et des coliques violentes. A la phase terminale. l'atteinte des centres nerveux supérieurs est manifeste.

Le médicament. aux doses thérapeutiques. est sans inconvénient pour les femelles gestantes et les agneaux de lait.

\section{REMERCIEMENTS}

Nous tenons à remercier vivement la Société Rhône-Poulenc pour sa contribution à la réalisation de ces essais, en particulier Mrs, QUENTIN et SIRY, Docteurs vétérinaires de la Direction scientifique.

Remis pour publication le 14 avril 1966. 


\section{SUMMARY}

Action of a new anthelminthic drug, the Tetramisole (16535 R. P.) on some helminthes of the sheep in Chad Republic

The anthelminthic power of the Tetramisole, a new drug which is derived from Imidazole $(16,535$ R. P.) was studied in 259 sheep in Chad Republic.

Althoug this drugis completely inactive on the Trematodes and Cestodes of the digestive system, doses of $40 \mathrm{mg} / \mathrm{kg}$ per os, or $12 \mathrm{mg} / \mathrm{kg}$ by sub-cutaneous injection (in 10 p. 100) solution) are able to insure almost completely the destruction of the following species: Gaigerio pochyscelis, Hoemoncus contortus, Desophagostomum colombionum, mature adults or immature. This drug is not so active on adult or immature Strongyloides. Its efficacily on Buckleyuris ovis and Buckleyris globulosa seems to be irregular, but better than that of Thiabendazole. Most of the $\mathrm{L}_{4}$ intranoduiar larvae of Oesophagostomum columbianum resist to the treatment, at any dose.

The Tetramisole, which is working as a nematodifuge, has proved to be of economic value for this reason that on the field the weight of the animals shows an increase of about 10,9-13,7 p. 100 after five weeks. The chimiotherapeutic rate ranges from 4,5 to 5 per os, and from 5 to 5,4 by subcutaneousway. The toxicity of 16,535 R. P. for the chadian sheep is therefore greater than the toxicity of Thiabendazole. At therapeutic doses, the tolerance of the pregnant females and of the sucking lambs is satisfactory.

\section{RESUMEN}

Acción de un nuevo antihelmíntico, el Tetramisole (16.535 R. P.) en algunos heimintos de la oveja en la República del Chad

El autar estudia el poder antihelmíntico de un nuevo derivado del Imidazole el Tetramisole (16.535 R. P.) en 259 ovinos de la República del Chad. Este medicamento es totalmente inactivo en los trematodos y los céstodos ; en cambio dosis de $40 \mathrm{mg} / \mathrm{kg}$ per os o de $12 \mathrm{mg} / \mathrm{kg}$ por inyección subcutánea (en solución a 10 por 100) permiten destruir casi completamente los adultos maduros o inmaduros de las especies siguientes: Gaigeria pachyscelis, Haemonchus confortus, Oesophagostomum columbianum.

Este antihelmíntico es menos activo en los estrongiloides adultos o inmaduros. Su actividad parece irregular en Buckleyuris ovis y en Buckleyuris globuloso, pero superior a la del Thiabendazole. Muchas larvas $L_{\mathbf{4}}$ intranodulares del Oesophagostamum columbianum resisten al tratamiento, cualquiera que sea la dosis utilizada.

El Tetramisole, que obra como un nematodifugo, tiene una importanciaeconómica indubitada ya que las ganancias de peso de los animales llegan a 10,913,7 por 100 sobre terreno al cabo de cinco semanas El. coeficiente quimioterapeôtico varia de 4,5 a 5 per os y de 5 a 5,4 por inyección subcutánea. La toxicidad de! 16.535 R. P. es superior a la del Thiabendazole para la oveja del Chad.

En dosis terapeúticas, la tolerancia de las hembras gravidas y de los corderos recentales es satisfactoria, 


\section{BIBLIOGRAPHIE}

1. GRABER (M.) et RECEVEUR (P.). - Parasitisme interne du mouton en zone sahélienne. Fsophagostomose nodulaire en particulier. Rev. elev. Med. Vet. Pays Trop., 1956, 9, 1, 5-20.

2. GRABER $\left(M_{1}\right)$. - Etude dans certaines conditions africaines de l'action antiparasitaire du Thiabendazole sur divers Helminthes des animaux domestiques. Helminthes du zébu. Rev. elev. med. Vet. Pays Trop., 1965 a, 18, 1. 39-58.

3. GRABER (M.). - Helminthes et Helminthiases faisant obstacle à l'amélioration de la production ovine en République du Tchad. Monographie I. E. M. V. P. T. et Lab. Forcha, 1965 b, 158 pp, 38 cartes, $25 \mathrm{gr}$.

4. GRABER (M.). - Le parasitisme des animaux domestiques en République du Tchad, 1966, 32 pp., 11 cartes.

5. GRABER (M.). - Etude dans certaines conditions africaines de l'action antiparasitaire du Thiabendazole sur divers Helminthes des animaux domestiques: II Helminthes du mouton, 1966 (d̀ paraître).

6. MAROTEL (G.) - Bull. Soc. Sci. Vet. Lyon, $1908,11,322$.

7. THIENPONT (D.), VANPARIJS (O. F. $1_{1}$ ), RAEMAEKERS (A., H. M.), VANDENBERK (J.), DEMOEN (J. A.), ALLEWIJN (F. T. N.), MARSBOOM (R. P. H.), NIEMEGEERS (C. J. E.), SCHELLEKENS (K. H. L.) AND JANS. SEN (A. J.). -- Tetramisole (R. 8299), a new potent broad spectrum anthelmintic. Noture, 1966, 209, 1084-86.

8. VEGLIA (F.). - Preliminary notes on the life-history of Esophagostomum columbianum. Ninth-Tenth Rep. Dir. Vet. Res. S. ofr., 1923, 810-823.

9. WALLEY (J. K.). - Tetramisole (Nilverm) in the treatment of gastro-intestinal worms and lungworms in domestic animals. Vet. Record, 1966, 78, 406-414. 